\title{
Explaining the extended $\mathrm{GeV}$ gamma-ray emission adjacent to HESS J1825-137
}

\author{
T. Collins, ${ }^{1 \star}$, G. Rowell ${ }^{1}$, A.M.W. Mitchell ${ }^{2}$, F. Voisin ${ }^{1}$, Y. Fukui ${ }^{3}$, H. Sano ${ }^{3}$, \\ R. Alsulami ${ }^{1,4}$ and $\mathrm{S}$. Einecke ${ }^{1}$ \\ ${ }^{1}$ School of Physical Sciences, University of Adelaide, Adelaide 5005, Australia \\ ${ }^{2}$ Department of Physics, ETH Zurich, CH-8093 Zurich, Switzerland \\ ${ }^{3}$ Department of Physics, University of Nagoya, Furo-cho, Chikusa-ku, Nagoya, 464-8601, Japan \\ ${ }^{4}$ Astronomy Dept, Faculty of Science, King Abdulaziz University, Jeddah, Saudi Arabia
}

Accepted XXX. Received YYY; in original form ZZZ

\begin{abstract}
HESS J1825-137 is one of the most powerful and luminous TeV gamma-ray pulsar wind nebulae (PWN). To the south of HESS J1825-137, Fermi-LAT observation revealed a new region of $\mathrm{GeV}$ gamma-ray emission with three apparent peaks (termed here, GeV-ABC). This study presents interstellar medium (ISM) data and spectral energy distribution (SED) modelling towards the $\mathrm{GeV}$ emission to understand the underlying particle acceleration. We considered several particle accelerator scenarios - the PWN associated with HESS J1825-137, the progenitor SNR also associated with HESS J1825-137, plus the gamma-ray binary system LS 5039. It was found that the progenitor SNR of HESS J1825-137 has insufficient energetics to account for all $\mathrm{GeV}$ emission. GeV-ABC may be a reflection of an earlier epoch in the history of the PWN associated with HESS 1825-137, assuming fast diffusion perhaps including advection. LS 5039 cannot meet the required energetics to be the source of particle acceleration. A combination of HESS J1825-137 and LS 5039 could be plausible sources.
\end{abstract}

Key words: gamma rays: ISM - ISM: individual (HESS J1825-137) - ISM: individual (LS 5039) - ISM: supernova remnants - ISM: clouds - cosmic-rays

\section{INTRODUCTION}

HESS J1825-137 is a luminous pulsar wind nebula (PWN) powered by the pulsar PSR J1826-1334 with spin down power of $\dot{E}=2.8 \times$ $10^{36} \mathrm{erg} \mathrm{s}^{-1}$ and characteristic age of $21.4 \mathrm{kyr}$ (Aharonian et al. 2006; Manchester et al. 2005). To the south of HESS J1825-137 a new region of $\mathrm{GeV}$ gamma-ray emission was revealed by Araya et al. (2019) using Fermi-LAT data (see Fig. 1). Araya et al. (2019) also suggested that this new region of $\mathrm{GeV}$ emission may be either an extension of HESS J1825-137 or unrelated to the system. If related to HESS J1825-137, the gamma-rays may be resultant from high energy particles from the PWN (in the form of electrons and positrons) or from the progenitor supernova remnant (SNR) linked to HESS J1825-137. If unrelated to HESS J1825-137 another source of high energy particles must exist towards this region. Araya et al. (2019) conducted spectral analysis in range $10-250 \mathrm{GeV}$ and fitted the spectra observed from this new region to a power-law $\left(\frac{\mathrm{d} N}{\mathrm{~d} E} \propto\right.$ $E^{-\Gamma}$ ) with index $\Gamma=1.92 \pm 0.07_{\text {stat }} \pm 0.05_{\text {sys. }}$. Fig. 3 from Araya et al. (2019) shows a TS map towards this region with three distinct peaks. We label the three GeV features GeV-A, GeV-B and GeV-C and are

^ E-mail: tiffany.collins@ adelaide.edu.au located at positions RA: $18^{\mathrm{h}} 29^{\mathrm{m}} 36.0^{\mathrm{s}}$, Dec: $-14^{\circ} 23^{\prime} 41.6^{\prime \prime}$, RA: $18^{\mathrm{h}} 30^{\mathrm{m}} 10.6^{\mathrm{s}}$, Dec: $-15^{\circ} 19^{\prime} 03.4^{\prime \prime}$ and RA: $18^{\mathrm{h}} 30^{\mathrm{m}} 21.4^{\mathrm{s}}$, Dec: $-16^{\circ} 00^{\prime} 40.3^{\prime \prime}$ respectively. Hereafter, the extended region of $\mathrm{GeV}$ emission will be referred to as $\mathrm{GeV}-\mathrm{ABC}$ for simplicity. Fig. 1 shows the locations of GeV-ABC relative to HESS J1825-137.

A TeV halo may be associated with HESS J1825-137 (Liu \& Yan 2020). TeV halos occur when electrons and positrons escape the PWN through diffusion and interact with the ambient interstellar medium producing surrounding $\mathrm{TeV}$ emission forming a 'halo'. The equivalent HAWC observatory source, eHWC J1825-134, has detected an extension of $0.36^{\circ}$ above $56 \mathrm{TeV}$ HAWC Collaboration et al. (2019). The extension around HESS J1825-137 can be seen to decrease with energy as shown by Fermi-LAT data Principe et al. (2019). It is possible that GeV-ABC may be an extension of the $\mathrm{TeV}$ emission around HESS J1825-137

A $\mathrm{H} \alpha$ rim like structure has been noted $120 \mathrm{pc}$ to the south east of the pulsar from PSR J1826-1334 (Voisin et al. 2016). This structure may be associated with the progenitor supernova remnant (SNR) that is linked to PSR J1826-1334. The $\mathrm{H} \alpha$ region overlays the Fermi-LAT GeV emission.

Another potential accelerator also towards $\mathrm{GeV}-\mathrm{ABC}$ is the gamma-ray binary system LS 5039, comprising a compact object and a massive O-type star 


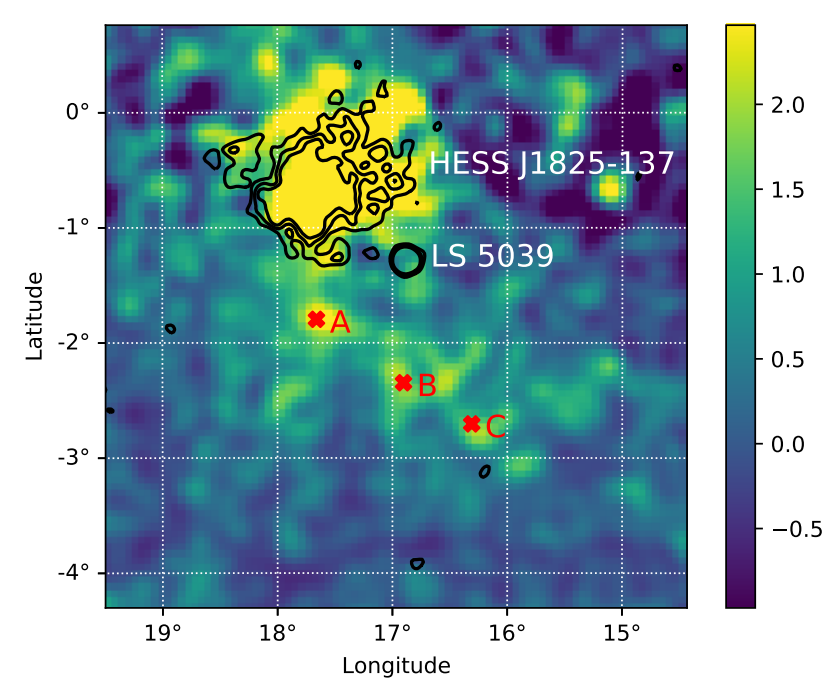

Figure 1. Fermi-LAT count map above $10 \mathrm{GeV}$ (Araya et al. 2019) towards HESS J1825-137 overlaid by black HESS significance contours at $1 \sigma, 2 \sigma$ and $3 \sigma$ (H. E. S. S. Collaboration et al. 2018).

This study presents gas analysis $(\mathrm{CO}, \mathrm{HI}$ and $\mathrm{H} \alpha$ ) towards the new region of Fermi-LAT GeV emission. To identify the origin of the $\mathrm{GeV}$ emission, spectral energy distribution (SED) modelling of the gamma-ray emission is performed assuming hadronic or leptonic particle populations accelerated by continuous or impulsive particle injectors.

\section{GAS MORPHOLOGY TOWARDS GEV-ABC}

PSR J1826-1334 and LS-5039, located within the vicinity towards $\mathrm{GeV}-\mathrm{ABC}$, are possible particle accelerators to produce the $\mathrm{GeV}$ emission as seen by Fermi-LAT. PSR J1826-1334 has measured dispersion distance of $3.9 \pm 0.4 \mathrm{kpc}$ (Taylor \& Cordes 1993) while the binary system LS-5039 distance is estimated to be $2.54 \pm 0.04 \mathrm{kpc}$ (Casares et al. 2005). For these two reasons ISM data in the velocity range of $15-30 \mathrm{~km} \mathrm{~s}^{-1}$ and $40-60 \mathrm{~km} \mathrm{~s}^{-1}$ corresponding to distances $1.6-2.8 \mathrm{kpc}$ and $3.5-4.5 \mathrm{kpc}$ respectively will be examined (Brand \& Blitz 1993).

\subsection{CO data}

Using the Nanten $12 \mathrm{CO}(1-0)$ survey data, the molecular hydrogen column density will be traced using conversion factor $N_{H_{2}}=X_{\mathrm{CO}} W_{12 C O}$. The $X_{\mathrm{CO}}$ factor is assumed to be constant $\approx 1.5 \times 10^{20} \mathrm{~cm}^{-2} \mathrm{~K}^{-1} \mathrm{~km}^{-1} \mathrm{~s}$ (Strong et al. 2004), over the galactic plane but may vary with galactocentric radius.

The top panel in Fig. 2 shows the $12 \mathrm{CO}(1-0)$ integrated intensity between $15-30 \mathrm{~km} \mathrm{~s}^{-1}$. Regions of clumpy gas are noted to the north east of HESS J1825-137 as noted by Voisin et al. (2016). Towards GeV-B, a region of denser gas are noticed which does not appear in the $40-60 \mathrm{~km} \mathrm{~s}^{-1}$ range as shown in the bottom panel of Fig. 2. In both velocity ranges the region towards GeV-ABC has relatively little gas compared to the galactic plane. The distance to these clouds is determined from the galactic rotation curve. Individual gas motion may give a false interpretation of the velocity range. In (Brand \& Blitz 1993) it was noticed that residuals of the modelled vs observed galactic rotation curve can be as great as $40 \mathrm{~km} \mathrm{~s}^{-1}$ with the average being around $12.8 \mathrm{~km} \mathrm{~s}^{-1}$.
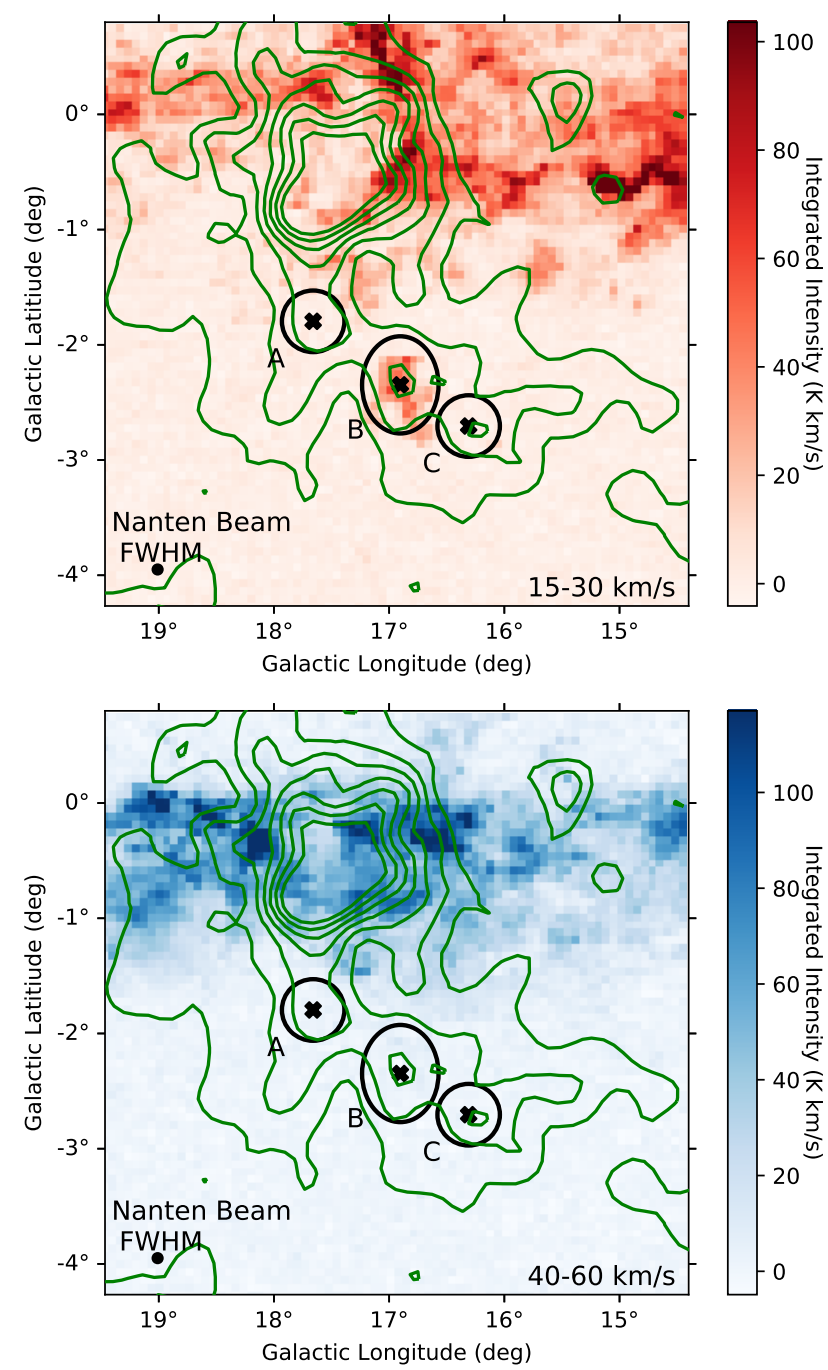

Figure 2. Nanten $12 \mathrm{CO}(1-0)$ integrated intensity in velocity ranges 15 $30 \mathrm{~km} \mathrm{~s}^{-1}$ (top) and $40-60 \mathrm{~km} \mathrm{~s}^{-1}$ (bottom). Green contours represents $\mathrm{GeV}$ emission as seen by Fermi-LAT at $1 \sigma$ to $7 \sigma$. The new regions of $\mathrm{GeV}$ emission, GeV-A, GeV-B and GeV-C, are shown by black markers. The Nanten beam size, shown in bottom left, is 2.6' (Mizuno \& Fukui 2004).

The mass of a cloud with average column density $N_{H_{2}}$ and cloud area $A$ can be calculated by:

$M_{H}=2.8 N_{H_{2}} A \frac{m_{p}}{m_{\odot}} M_{\odot}$

where $M_{H}=2.8 M_{H_{2}}$ includes a $20 \%$ He component. The cloud areas used can be seen in Fig. 2 The number density can then be obtained through:

$n_{H}=\frac{M_{H}}{4 / 3 \pi R^{3} m_{p}} \mathrm{~cm}^{-3}$

where $\mathrm{R}$ represents the radius of cloud area considered. The results of these calculations are given in Table A1 with cloud areas shown in Fig. 2. The size and shape of object B is chosen to contain dense gas seen in the $15-30 \mathrm{~km} \mathrm{~s}^{-1}$ velocity ranges, while object $\mathrm{A}$ and $\mathrm{C}$ were chosen to be the same size but independent of object B. The clumps seen towards GeV-B in the top panel of Fig. 2 is an 

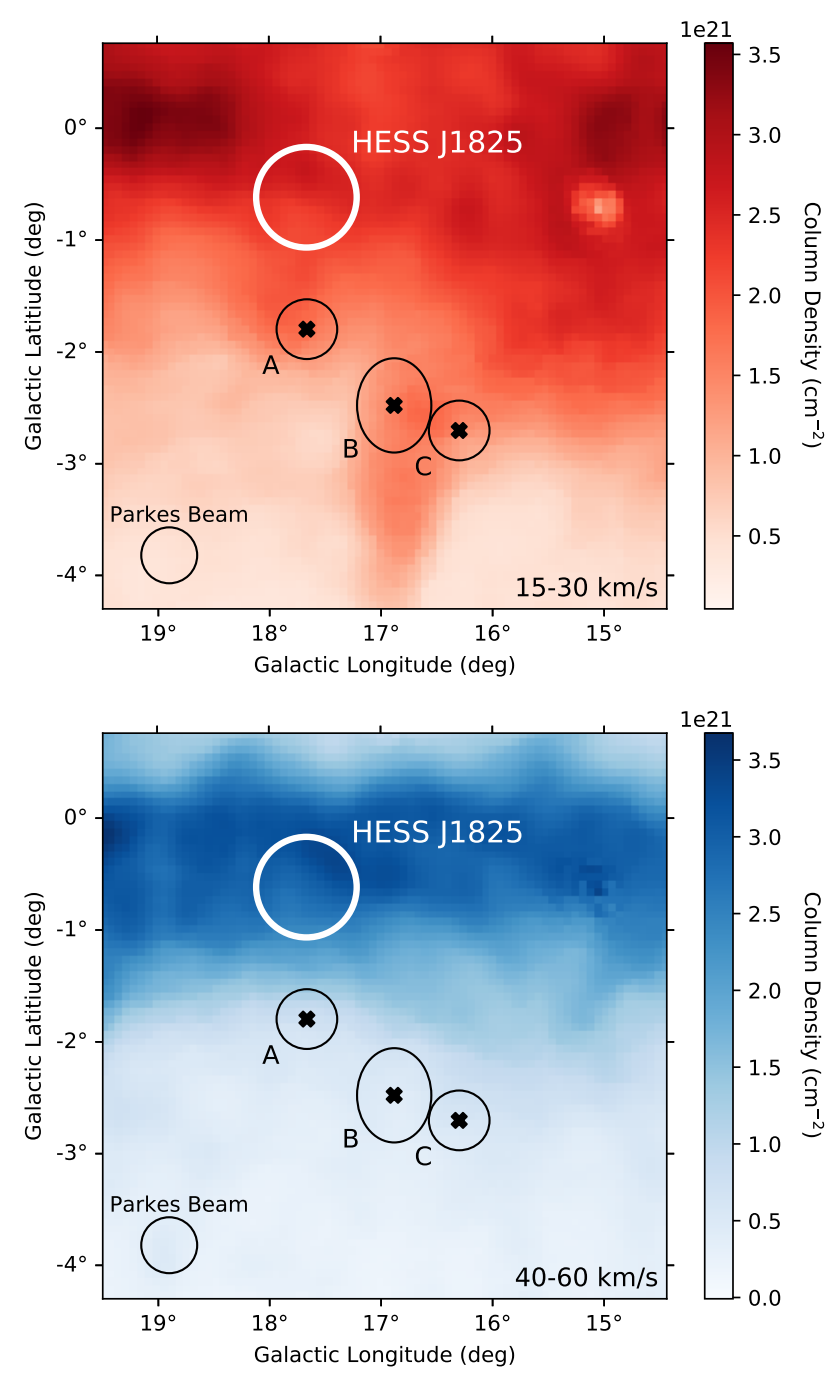

Figure 3. Parkes HI integrated column density (from GASS) in velocity ranges $15-30 \mathrm{~km} \mathrm{~s}^{-1}$ (top) and $40-60 \mathrm{~km} \mathrm{~s}^{-1}$ (bottom) (McClureGriffiths et al. 2009). The regions that were analysed here can be seen in white (HESS J1825-137 ) and black (new GeV regions). The Parkes beam size, shown in bottom left, is $15^{\prime}$ See Table A2 for results.

order of magnitude denser compared to the ISM towards GeV-A and $\mathrm{GeV}-\mathrm{C}$.

\subsection{HI data}

The Galactic All Sky Survey of atomic Hydrogen (HI) data set will be used to trace atomic hydrogen towards new region of $\mathrm{GeV}$ gamma-ray activity (McClure-Griffiths et al. 2009).

The integrated column density in the velocity of ranges of interest can be seen in Fig. 3. In the $40-60 \mathrm{~km} \mathrm{~s}^{-1}$ range towards the region around $\mathrm{GeV}-\mathrm{ABC}$, the $\mathrm{HI}$ column density is relatively low compared to the galactic plane. The area towards the new $\mathrm{GeV}$ emission has slightly greater $\mathrm{HI}$ density in the $15-30 \mathrm{~km} \mathrm{~s}^{-1}$ velocity range compared to the $40-60 \mathrm{~km} \mathrm{~s}^{-1}$ velocity range.

The calculated HI parameters for different regions towards HESS J1825-137 and the new emission of GeV gamma-rays can be seen in Table A2. Atomic hydrogen, compared to molecular hydrogen, is less abundant. The total ISM parameters are shown in Table 1. The contribution of atomic hydrogen compared to molec-
Table 1. Total ISM densities for HESS J1825-137 and new GeV emission regions $\mathrm{GeV}-\mathrm{A}, \mathrm{GeV}-\mathrm{B}$ and $\mathrm{GeV}-\mathrm{C}$.

\begin{tabular}{cccc}
\hline $15-30 \mathrm{~km} \mathrm{~s}^{-1}$ & Object & $\boldsymbol{M}_{\boldsymbol{H}}\left(\boldsymbol{M}_{\odot}\right)$ & $n_{\boldsymbol{H}}\left(\mathrm{cm}^{-3}\right)$ \\
\hline & HESS J1825-137 & $1.18 \times 10^{5}$ & 40.1 \\
GeV-A & $4.56 \times 10^{3}$ & 7.2 \\
& GeV-B & $1.38 \times 10^{5}$ & 79.8 \\
& GeV-C & $2.30 \times 10^{3}$ & 3.6 \\
\hline $40-60 \mathrm{~km} \mathrm{~s}^{-1}$ & Object & $\boldsymbol{M}_{\boldsymbol{H}}\left(\boldsymbol{M}_{\odot}\right)$ & $n_{\boldsymbol{H}}\left(\mathrm{cm}^{-3}\right)$ \\
\hline & HESS J1825-137 & $5.22 \times 10^{5}$ & 178 \\
& GeV-A & $8.93 \times 10^{3}$ & 14 \\
GeV-B & $1.26 \times 10^{4}$ & 7.3 \\
& GeV-C & $3.11 \times 10^{2}$ & 0.5 \\
\hline
\end{tabular}

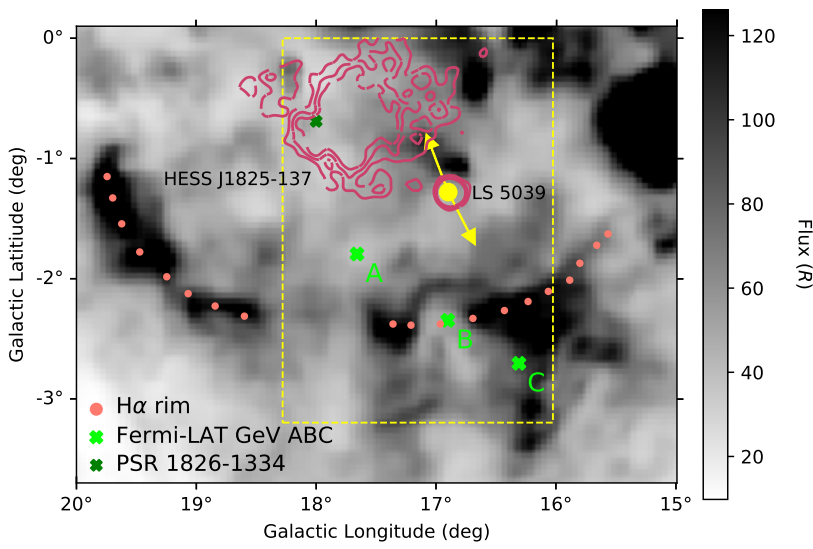

Figure 4. $\mathrm{H} \alpha$ emission from the FWHM survey towards HESS J1825-137 and surrounding regions (Finkbeiner 2003). HESS J1825-137 can be seen by $\sigma=1,2$ and 3 purple TeV contours with PSR J1826-1334 represented by the dark green cross and LS-5039 by the yellow dot lying to the lower right with yellow radio jets described by Paredes et al. (2002). GeV regions $\mathrm{GeV}-\mathrm{A}, \mathrm{GeV}-\mathrm{B}$ and $\mathrm{GeV}-\mathrm{C}$ can be seen as green neon crosses. The $\mathrm{H} \alpha$ rims noted by Stupar et al. (2008) and Voisin et al. (2016) are shown by pink dots. A closer look at the region contained within yellow box is shown in Fig. 5.

ular hydrogen is minimal (approximately 10\%) to the total density of hydrogen gas.

\section{$2.3 \quad \mathrm{H} \alpha$ data}

An intensity map of $\mathrm{H} \alpha$ emission towards HESS J1825-137 and surrounding regions can be seen in Fig. 4 from the FWHM survey (Finkbeiner 2003). The $\mathrm{H} \alpha$ rims detected by Stupar et al. (2008) and (Voisin et al. 2016) can clearly be seen and are located $\approx 120 \mathrm{pc}$ from PSR J1826-1334 if it lies at the same distance $(3.9 \mathrm{kpc})$ as the pulsar. From hydrodynamical simulations, the supernova remnant radius is, at least, four times the radius of this PWN (van der Swaluw $\& \mathrm{Wu} 2001$ ); this suggests a SNR radius of $140 \mathrm{pc}$ as calculated by Voisin et al. (2016) agreeing with the rim of ionized gas seen in Fig. 4 and predictions made by de Jager \& Djannati-Ataï (2009).

Overlaying combined molecular and $\mathrm{HI}$ contours onto the $\mathrm{H} \alpha$ map, (see Fig. 5), it can be seen the $\mathrm{CO}(1-0)$ cloud in the $15-$ $30 \mathrm{~km} \mathrm{~s}^{-1}$ range noted in section 2.1 overlaps a region of reduced $\mathrm{H} \alpha$ emission. This may indicate that the cloud is in the foreground or that the $\mathrm{CO}(1-0)$ cloud is surrounded by $\mathrm{H} \alpha$ gas.

Two different methods were utilised to calculate the density of ionised hydrogen towards the regions of interest. The details of these calculations are provided in appendix B. The results of both methods are shown in Table A3. Method A assumes that the 


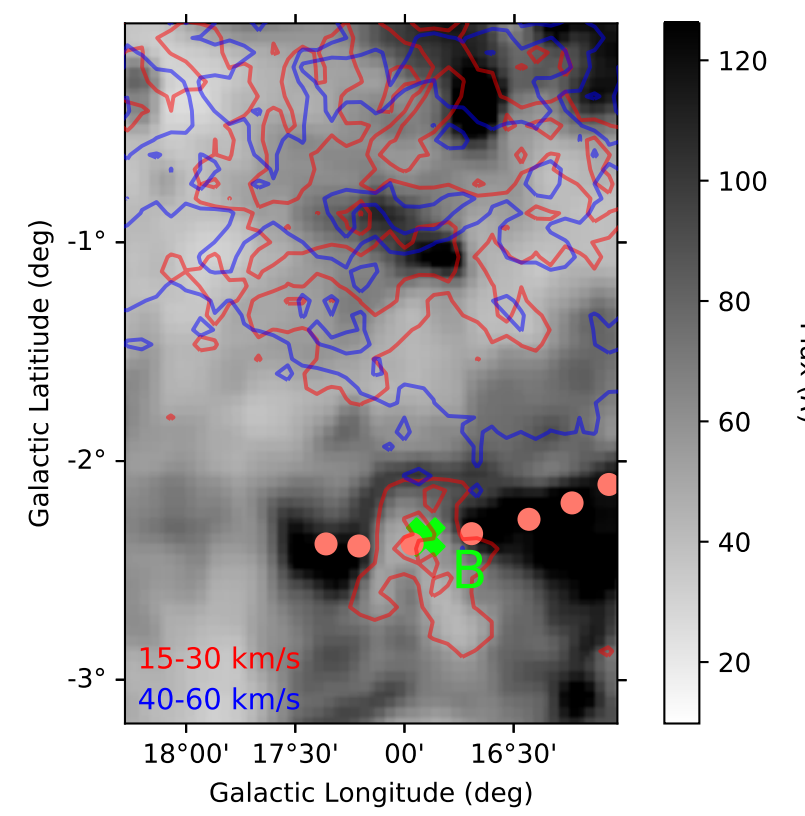

Figure 5. Zoomed in $\mathrm{H} \alpha$ intensity overlayed with Nanten $12 \mathrm{CO}(1-0)$ in the $15-30 \mathrm{~km} \mathrm{~s}^{-1}$ (red) and $40-60 \mathrm{~km} \mathrm{~s}^{-1}$ (blue) range. The $\mathrm{H} \alpha$ rims are indicated by the pink dots. Note that this corresponds to the yellow box in Fig. 4. The dense $\mathrm{CO}$ cloud in the $15-30 \mathrm{~km} \mathrm{~s}^{-1}$ velocity range towards $\mathrm{GeV}-\mathrm{B}$ can be seen to anti-correlate with the $\mathrm{H} \alpha$ emission.

density of photons is approximately equal to the density of ionised gas, assuming that atoms are not re-excited by an external source. Method B considers basic radiation transfer. It is expected that the ratio of ionised to neutral hydrogen atoms is $\approx 10^{-6}$ which agrees with both methods (Draine 2011). Therefore ionised hydrogen does not significantly contribute to the total density of the ISM.

\section{PARTICLE TRANSPORT}

After having mapped out the ISM, we can now consider the diffusive transport of high energy particles. In this study the spectral energy distribution modelling assumes that the high energy particles are able to enter GeV-ABC in sufficient number and energy range to produce the $\mathrm{GeV}$ gamma radiation. This section will look into the validity of this assumption assuming a purely diffusive scenario and looking at the cooling time of particles and how it affects the particle transport.

Once high energy particles are emitted by the PWN (or progenitor SNR) they must traverse the interstellar medium before entering the region towards $\mathrm{GeV}-\mathrm{ABC}$. In a purely diffusive scenario, the distance that particles of energy $E$ diffuse into the ISM in time $t$ is estimated by

$R(E, t)=\sqrt{2 D(E, B) t} \quad[\mathrm{~cm}]$

where

$D(E, B)=\chi D_{0} \sqrt{\frac{E / \mathrm{TeV}}{B / 3 \mu \mathrm{G}}} \quad\left[\mathrm{cm}^{2} \mathrm{~s}^{-1}\right]$

$D_{0}=1 \times 10^{29} \mathrm{~cm}^{2} \mathrm{~s}^{-1}$ is the galactic diffusion coefficient at $1 \mathrm{TeV}$ and $\chi$ takes values of around 0.01 (with variation) (Berezinskii et al. 1990; Gabici et al. 2007). As particles traverse the ISM they

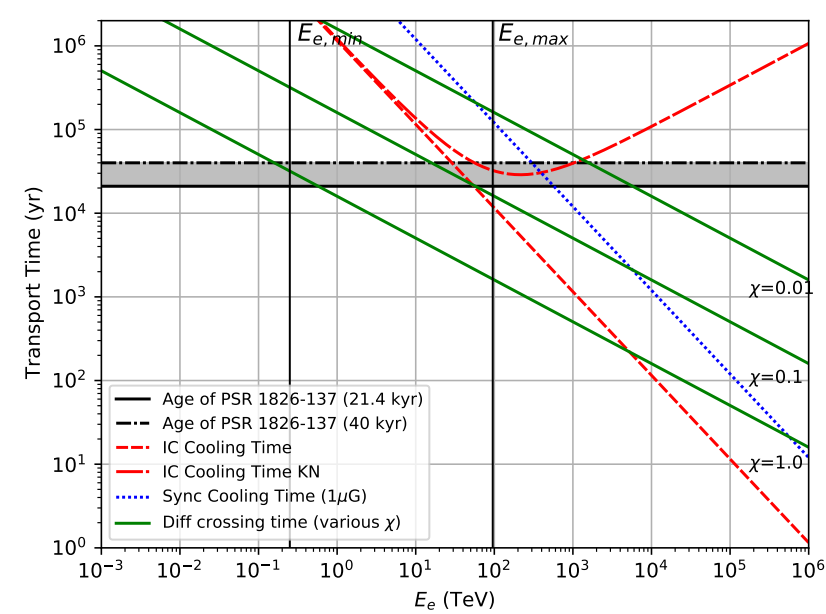

Figure 6. Transport time for particles to traverse from PSR 1826-1336 to GeV-B versus cooling time of synchrotron and IC processes. The ambient density of the ISM is assumed to be $n=1 \mathrm{~cm}^{-3}$. The black horizontal solid and dashed lines show the two possible ages of PSR J1826-1334 ( $t=21.4 \mathrm{yr}$ and $t=40 \mathrm{yr}$ respectively), the dotted blue line shows the cooling time through synchrotron losses at $1 \mu \mathrm{G}$ while the two red dashed lines is through IC losses in the Thompson and Klein Nishina regime. The solid lines with varying $\chi$ values shows the values necessary for particles with that energy to reach GeV-B in the available time through ISM with magnetic field $B=1 \mu \mathrm{G}$. The inferred minimum and maximum electron energy, $E_{\mathrm{e}}$, min and $E_{\mathrm{e}, \max }$, emitted by the pulsar wind nebula is shown by the vertical solid lines.

suffer energy losses through IC, bremsstrahlung and synchrotron radiation. The cooling time for bremsstrahlung, $t_{\text {brem }}$, Inverse Compton, $t_{\mathrm{IC}}$, and synchrotron, $t_{\mathrm{sync}}$, loss processes is given by:

$$
\begin{aligned}
t_{\mathrm{brem}} & \approx \frac{4 \times 10^{7}}{n \mathrm{~cm}^{-3}} \quad \mathrm{yr} \\
t_{\mathrm{IC}} & \approx\left\{\begin{array}{lll}
3 \times 10^{5} U^{-1}\left(\frac{E}{\mathrm{TeV}}\right)^{-1} \text { yr } \\
3.1 \times 10^{5} U^{-1}\left(\frac{E}{\mathrm{TeV}}\right)^{-1} f_{\mathrm{KN}}^{-1} & \text { yr } \quad \text { KN Regime }
\end{array}\right. \\
t_{\mathrm{sync}} & \approx 12 \times 10^{6}\left(\frac{B}{\mu \mathrm{G}}\right)^{-2}\left(\frac{E}{\mathrm{TeV}}\right)^{-1} \mathrm{yr}
\end{aligned}
$$

where $U=0.26 \mathrm{eV} \mathrm{cm}^{-3}$ is the energy density of the cosmic microwave background and $f_{\mathrm{KN}}$ is the Klein Nishina $(\mathrm{KN})$ suppression factor given by Moderski et al. (2005):

$f_{\mathrm{KN}}=\left(1+40 \frac{E}{\mathrm{TeV}} k T_{\mathrm{eV}}\right)^{-1.5}$

for an electron with energy $E$ interacting with photon field with temperature $T$ (with $k T$ in units of $\mathrm{eV}$ ). If the density of the ISM is $n=1$, the time it takes for particles of varying energies to be emitted by the PWN and travel to GeV-B ( $\approx 70 \mathrm{pc})$ and the cooling time is shown in Fig. 6. The intersection of the diffusion time and the age of PSR J1826-1334 represents the minimum particle energy that can reach GeV-B. Naturally if the pulsar is older, more lower energy particles can reach GeV-B. The maximum energy of electrons able to reach GeV-B is found through the intersection of the diffusion line and the IC cooling time (the quickest process where electrons lose most of their energy).

In IC processes, the final energy of the photon, $E_{\Gamma, \mathrm{TeV}}$, is 
related to initial electron energy, $E_{e}, \mathrm{TeV}$ and initial photon energy $E_{i, \mathrm{eV}}$ through:

$$
E_{\Gamma, \mathrm{TeV}}=E_{e, \mathrm{TeV}} \frac{h}{\left(1+h^{4 / 5}\right)^{5 / 4}}
$$

where $h \approx 31.5 E_{e, \mathrm{TeV}} E_{i, \mathrm{eV}}$ (Hinton \& Hofmann 2009). Photons up to $54 \mathrm{TeV}$ has been observed towards HESS J1825-137 (H. E. S. S. Collaboration et al. 2019). Assuming IC interactions with the cosmic microwave background are responsible for this emission, this is equivalent to an electron with maximum energy of $96 \mathrm{TeV}$. Similarly photons as low as $1.26 \mathrm{GeV}$ has been observed by Fermi-LAT (Principe et al. 2020), which is equivalent to minimum electron energy of $0.25 \mathrm{TeV}$. This electron energy range is shown by the vertical lines in figure 6 . This further limits how many electrons are able to diffuse to GeV-B.

The region around the PWN can harbour a strong magnetic field strength compared the surrounding ISM. Eq. $5 \mathrm{c}$ outlines the cooling time for the synchrotron processes as a function of electron energy and magnetic field; (as shown by Fig. 6). Consequently electrons in the zone around the pulsar wind nebula will experience stronger synchrotron losses compared to what is shown in Fig. 6.

\section{SED MODELLING OF THE GAMMA-RAY EMISSION}

Two main pathways are possible for the production of $\mathrm{GeV}$ gamma radiation. In a hadronic scenario, proton-proton interactions with the ISM leading to the production of neutral pions which, in turn, decay into gamma-radiation. Leptonic scenarios include synchrotron emission associated with the magnetic field pervading in the ISM, inverse-compton emission with the cosmic microwave background and Bremsstrahlung interactions with the ISM.

Two types of particle accelerators will be considered; continuous and impulsive accelerators. Continuous accelerators constantly inject particles into the interstellar medium throughout their lifetime. For this study, it will be assumed that particles will be injected at a constant energy rate. Continuous accelerators may include pulsars and stellar clusters for example. On the other hand an impulsive accelerator, such as a supernova remnant, injected particles in one big burst in the past.

The particles that are injected are then free to undergo interactions producing radio to gamma-ray emission. The following section will describe potential particle accelerators that may result in the $\mathrm{GeV}$ gamma radiation as seen by Fermi-LAT. The model utilised in this study takes the initial particle spectrum and then lets the system evolve over the age of the particle accelerator. After the allocated time has passed, the final particle spectrum is calculated and the gamma-ray spectrum is extracted. For further explanation of the process utilised in this study, please refer to Appendix C.

The ISM density of the region to be modelled will utilise the data calculated in section 2. In turn the magnetic field strength, which affects the production of synchrotron radiation, is related to the density of ISM through the relation (Crutcher 1999):

$B\left(n_{H}\right)=100 \sqrt{\frac{n_{H}}{10^{4} \mathrm{~cm}^{-3}}} \mu \mathrm{G}$

Note that the updated version of this relation provides a slightly higher magnetic field estimation (Crutcher et al. 2010). Crutcher's relation computes the maximum magnetic field in a molecular cloud, allowing estimations calculated using (Crutcher 1999) to be acceptable for this study.

\subsection{Potential Particle Accelerators}

\subsubsection{HESS J1825-137 (PWN - Continuous)}

As shown in Fig. 2, it appears the Fermi GeV-ABC might be an extension of HESS J1825-137.

A part of the spin down power of HESS J1825-137, $2.8 \times$ $10^{36} \mathrm{erg} \mathrm{s}^{-1}$, is channelled into accelerating particles that propagate out of the system. It was found that the major axis of gammaemission is to the south-west of the pulsar towards GeV-ABC (H. E. S. S. Collaboration et al. 2019). The asymmetry in the gamma-ray emission may indicate an asymmetry in the particle emission by the PWN. The PWN would be a continuous source of high energy electrons towards the new region of GeV Fermi-LAT emission.

\subsubsection{HESS J1825-137 Progenitor (SNR - Impulsive)}

Here we assume the progenitor SNR is an impulsive accelerator where the bulk of the cosmic rays escape the system very early and travel ahead of the SNR. Cosmic rays of energies $E$ escape the SNR in time $\chi$ :

$\chi(E)=t_{\operatorname{Sedov}}\left(\frac{E}{E_{\max }}\right)^{-1 / \delta}$

where $t_{\text {Sedov }}=200 \mathrm{yr}$ is the onset of the Sedov Phase of a SNR, $\delta=2.48$ is a parameter describing the energy dependent release of cosmic rays and $E_{\max }=500 \mathrm{TeV}$ is the maximum possible cosmic ray proton energy (Gabici et al. 2009). The TeV cosmic rays responsible for the emission of gamma-rays towards $\mathrm{GeV}-\mathrm{ABC}$ have an escape time of $\sim 2 \mathrm{kyr}$. This is negligible compared to age of the pulsar (21.4 kyr). The size of the SNR during the Sedov phase can be determined through (Reynolds 2008):

$R=0.31 E_{51}^{1 / 5}\left(\mu_{1} / 1.4\right)^{-1 / 5} n^{-1 / 5} t_{\mathrm{yr}}^{2 / 5} \mathrm{pc}$

where $E_{51}$ is the kinetic energy of the SNR in units of $10^{51} \mathrm{erg}$, $\mu_{1}$ is the mean mass per particle and $n$ is the background ISM density. If we assume $E_{51}=1, n=1 \mathrm{~cm}^{-3}$ and $\mu=1.41$; at age $2 \mathrm{kyr}$, the SNR will have a radius of $\sim 7$ pc. The TeV cosmicrays will escape the SNR at this radius and diffuse ahead of the SNR to GeV-ABC. Therefore the SNR progenitor associated with HESS J1825-137, as noted by Stupar et al. (2008) and Voisin et al. (2016), can be approximated as an impulsive source of high energy particles. Additionally, it is generally believed that $10-30 \%$ of the $10^{51}$ erg of kinetic energy released in a supernova is channelled into accelerated high energy particles by the subsequent supernova remnant.

The distance to the PWN and SNR associated with HESS J1825-137 will be assumed to be $3.9 \mathrm{kpc}$ (Taylor \& Cordes 1993). For this reason ISM parameters in the $40-60 \mathrm{~km} \mathrm{~s}^{-1}$ velocity range (see Table. 1) will be used in the SED modelling of GeV-A, GeV-B and GeV-C. Due to the anti-correlation of $\mathrm{CO}(1-0)$ to $\mathrm{H} \alpha$ emission as seen in Fig. 5, the dense gas towards GeV-B in the $15-30 \mathrm{~km} \mathrm{~s}^{-1}$ velocity will also be considered as a target for high energy particles to emit radiation. Due to individual gas motion compared to the galactic rotation curve (Brand \& Blitz 1993), this region of dense gas may be located at the same distance as HESS J1825-137. 


\subsubsection{LS 5039 (accretion powered - Continuous)}

LS 5039 is a microquasar and X-ray binary system (Motch et al. 1997). LS 5039 contains an O type star in orbit around an unknown compact object with mass $\approx 3.7 M_{\odot}$ (Ptuskin \& Zirakashvili 2005). This high mass is greater than standard neutron star masses leading to the possibility of the compact object being a black hole. The high mass of the compact object suggests that the progenitor was born in the binary system with a mass greater than the $\mathrm{O}$ type star $\left(M_{O}=22.9 M_{\odot}\right)$. The age of LS 5039 is unknown; the lifetime of an O-type star is of order a few million years, giving an upper limit to the age of the system. The minimum and maximum plausible ages, of $1 \times 10^{3}$ yrs and $1 \times 10^{6}$ yrs respectively, will be considered in the modelling (Moldón et al. 2012). Moldón et al. (2012) aimed to find the galactic trajectory of LS 5039 to determine its birthplace. Depending on where LS 5039 was born, Moldón et al. (2012) gives the age of the system to be between $0.1-1.2 \mathrm{Myr}$. Therefore an age of $10^{5}$ yr will also be considered in the SED modelling of this paper. In modelling the SED, these ages reflect the time when high energy particles enter GeV-ABC. Assuming diffusion is the particle transport method as in section 3 , the transportation time of high energy particles between LS 5039 and GeV-ABC $\left(\approx 10^{4}\right.$ yrs $)$ is negligible compared to the age of LS 5039.

After formation, the compact object associated with LS 5039 continuously accretes matter from its star companion allowing particles to be accelerated in a relativistic radio jet. This may be a continuous accelerator of particles to form the new $\mathrm{GeV}$ region as seen by Fermi-LAT. Radio jets described by Paredes et al. (2002) can be seen in figure 4 . The average accretion luminosity of LS 5039 was calculated by Casares et al. (2005) to be $L_{\mathrm{acc}}=8 \times 10^{35} \mathrm{erg} \mathrm{s}^{-1}$. The luminosity released in the vicinity of LS 5039 is given by: $L_{\text {radio, } 0.1-100 \mathrm{GHz}} \approx 1.3 \times 10^{31} \mathrm{erg} \mathrm{s}^{-1}$ (Marti et al. 1998), $L_{\text {X-ray, } 3-30 \mathrm{keV}}=0.5-5 \times 10^{34} \mathrm{erg} \mathrm{s}^{-1}$ (Bosch-Ramon et al. 2005) and $L_{>100 \mathrm{GeV}}=2.7 \times 10^{35} \mathrm{erg} \mathrm{s}^{-1}$ (Casares et al. 2005). Therefore (Casares et al. 2005) concluded that approximately one third of the accretion luminosity is channelled into the relativistic jets. The remaining $5.5 \times 10^{35} \mathrm{erg} \mathrm{s}^{-1}$ can be channelled into GeV-ABC. Given the distance estimate to LS 5039 of $2.5 \mathrm{kpc}$, the ISM within the $15-30 \mathrm{~km} \mathrm{~s}^{-1}$ regime will be considered. It has been noticed that the radio jets are persistent with variability on day, week and year time scales (Marcote et al. 2015).

\subsubsection{LS 5039 Progenitor (SNR - Impulsive)}

Whether the compact object within LS 5039 is a black hole or neutron star, the compact object is the result of a star gone supernova. By this logic an impulsive source of high energy particles occurred sometime in the past. At the time of writing, no clear SNR has been linked to LS 5039. If LS 5039 has age of order $10^{5} \mathrm{yrs}$, any SNR will be too old to be detected.

\subsection{Spectral Energy Distribution}

The spectral analysis conducted by Araya et al. (2019) towards GeV-ABC is shown in Fig. 7. They found that a simple powerlaw $\left(\frac{\mathrm{d} N}{\mathrm{~d} E} \propto E^{-\Gamma}\right)$ best describes the spectrum with spectral index of $\Gamma=1.92 \pm 0.07_{\text {stat }} \pm 0.05_{\text {sys }}$ and integrated flux of $\phi_{0}=\left(1.46 \pm 0.11_{\text {stat }} \pm 0.13_{\text {sys }}\right) \times 10^{-9}$ photons $/ \mathrm{cm}^{2} / \mathrm{s}$.

In the study by Araya et al. (2019), individual peaks GeV-A, $\mathrm{B}$ and $\mathrm{C}$ were found to have spectral indices $\Gamma_{A}=1.78 \pm 0.25_{\text {stat }}$, $\Gamma_{B}=1.7 \pm 0.4_{\text {stat }}$ and $\Gamma_{C}=1.43 \pm 0.23_{\text {stat }}$ respectively. The extended $\mathrm{GeV}$ emission observed by Fermi-LAT will be modelled

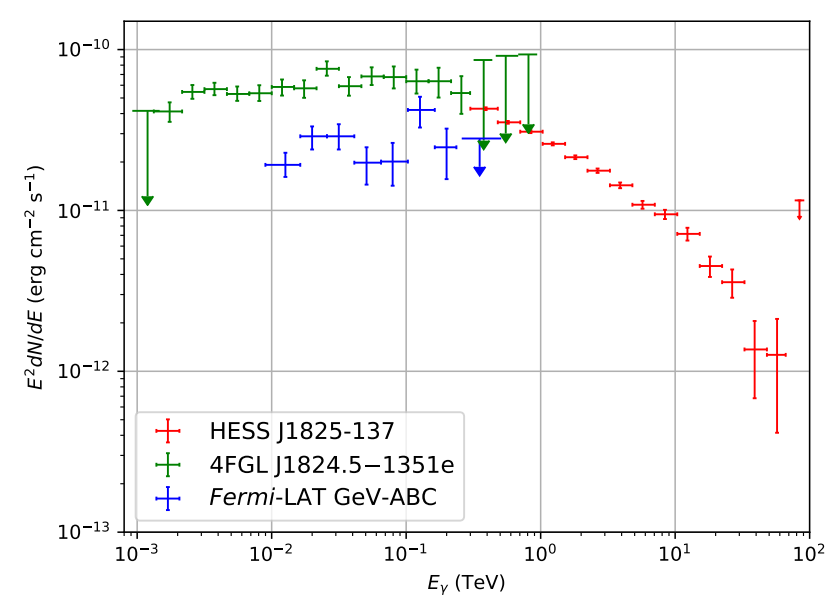

Figure 7. Spectral Energy Distribution of GeV-ABC as revealed by Araya et al. (2019) is shown in blue. The flux points of HESS J1825-137 are represented by red. H. E. S. S. Collaboration et al. (2019). Fermi-LAT data towards HESS J1825-137 (Principe et al. 2020) shown in green can be seen to follow the HESS data points as noticed by H. E. S. S. Collaboration et al. (2019).

by approximating the spectra of $\mathrm{GeV}-\mathrm{ABC}$ as coming from three sources corresponding to the peaks observed by Araya et al. (2019). By integrating the flux over all energy ranges for all three peaks and normalising to the spectra of $\mathrm{GeV}-\mathrm{ABC}$, the amount each peak contributes to the total flux can be determined. This assumes that the entirety of the $\mathrm{GeV}$ emission originates from the three peaks. As seen by Fig 3 from Araya et al. (2019) GeV A, B and C contains the majority of the $\mathrm{GeV}$ emission.. It was found that GeV-A, GeV-B and $\mathrm{GeV}-\mathrm{C}$, contributed $37 \%, 34 \%$ and $29 \%$ of the total $\mathrm{GeV}$ flux respectively. For each peak in the $\mathrm{GeV}$ gamma-ray emission region, spectral energy distributions based on different particles accelerators (e.g. PWN HESS J1825-137 and LS 5039) will be modelled and fit by eye to the data. Input parameters will also be varied to provide a range where the model matches the data. The ROSAT x-ray upper limit towards GeV-ABC was calculated using the ROSAT X-Ray Background Tool (Sabol \& Snowden 2019).

Input parameters of the spectral energy distribution modelling towards the new region of $\mathrm{GeV}$ emission can be seen in Tables 2 and 3 and Tables 4 and 5 for HESS J1825-137 and LS 5039 being the source of high energy particles respectively.

An example fit to the spectral energy distribution is shown in Fig. 8 . It is assumed that both hadronic and leptonic particles followed an exponential cutoff power law injection spectra $\left(\frac{\mathrm{d} N}{\mathrm{~d} E} \propto E^{-\Gamma} \exp \left(-E / E_{c}\right)\right)$. It is important to note that the energy budget/injection luminosity, $W$ or $\dot{W}$, that is inferred reflects the energy budget for each individual peak (GeV-A, GeV-B and GeV-C) and not the total energy budget/injection luminosity for the combination of all three regions.

Assuming constant cosmic ray density within a supernova remnant, the inferred energy $\left(W_{\mathrm{SNR}}\right)$ of the SNR can be calculated. The filling factor, $f_{f}$, is defined to be the ratio of the area of GeV-A, B or $\mathrm{C}$ to the projected area of the SNR. The inferred energy of the SNR is then given by:

$W_{\mathrm{SNR}}=\frac{W}{f_{f}}$ 
Table 2. SED model parameters matching the observed emission of GeV-A, B and C for a hadronic scenario. The particle accelerators considered are the impulsive progenitor SNR associated with PSR 1826-1334 and the continuous accelerator associated with the pulsar wind nebula, HESS J1825-137. High energy particles are assumed to be injected with a power law spectra with an exponential cutoff: $\frac{\mathrm{d} N}{\mathrm{~d} E} \propto E^{-\Gamma} \exp \left[E / E_{c}\right]$.

\begin{tabular}{|c|c|c|c|c|c|c|c|c|}
\hline \multirow{2}{*}{$\begin{array}{c}\text { Accelerator } \\
\text { PSR } 1826-1334 \text { or SNR }\end{array}$} & \multirow[b]{2}{*}{ Peak } & \multicolumn{5}{|c|}{ Hadronic } & \multirow[b]{2}{*}{${ }^{3} W_{\mathrm{SNR}}$ or ${ }^{4} \dot{W}_{\mathrm{tot}}$} & \\
\hline & & $n_{H}\left(\mathrm{~cm}^{-3}\right)$ & ${ }^{1} W$ or ${ }^{2} \dot{W}$ & & $\Gamma$ & $E_{C}(\mathrm{TeV})$ & & \\
\hline Impulsive (SNR) & $\mathrm{A}$ & 14 & $1.0 \times 10^{50}$ & erg & 2.0 & 50 & $6.0 \times 10^{51}$ & erg \\
\hline \multirow[t]{3}{*}{$t=21 \times 10^{3} \mathrm{yrs}$} & B & 79.8 & $1.5 \times 10^{49}$ & & 2.0 & 50 & $4.7 \times 10^{50}$ & \\
\hline & B & 7.0 & $1.5 \times 10^{50}$ & & 2.0 & 50 & $4.7 \times 10^{51}$ & \\
\hline & $\mathrm{C}$ & 1.0 & $1.2 \times 10^{51}$ & & 2.0 & 50 & $7.3 \times 10^{52}$ & \\
\hline Impulsive (SNR) & $\bar{A}$ & 14 & $1.0 \times 10^{50}$ & erg & 2.0 & 50 & $6.1 \times 10^{51}$ & erg \\
\hline \multirow[t]{3}{*}{$t=40 \times 10^{3} \mathrm{yrs}$} & B & 79.8 & $1.5 \times 10^{49}$ & & 2.0 & 50 & $4.7 \times 10^{50}$ & \\
\hline & B & 7.0 & $1.5 \times 10^{50}$ & & 2.0 & 50 & $4.7 \times 10^{51}$ & \\
\hline & $\mathrm{C}$ & 1.0 & $1.1 \times 10^{51}$ & & 2.0 & 50 & $6.7 \times 10^{52}$ & \\
\hline Continuous (PWN) & $\mathrm{A}$ & 14 & $1.2 \times 10^{38}$ & $\operatorname{erg~s}^{-1}$ & 2.0 & 50 & & \\
\hline \multirow[t]{3}{*}{$t=21 \times 10^{3} \mathrm{yrs}$} & B & 79.8 & $2.0 \times 10^{37}$ & & 2.0 & 50 & & \\
\hline & B & 7.0 & $2.2 \times 10^{38}$ & & 2.0 & 50 & & \\
\hline & $\mathrm{C}$ & 1.0 & $1.7 \times 10^{39}$ & & 2.0 & 50 & $1.8-2.0 \times 10^{39}$ & $\operatorname{ergs} s^{-1}$ \\
\hline Continuous (PWN) & $\mathrm{A}$ & 14 & $8.0 \times 10^{37}$ & $\operatorname{erg~s}^{-1}$ & 2.0 & 50 & & \\
\hline \multirow[t]{3}{*}{$t=40 \times 10^{3} \mathrm{yrs}$} & B & 79.8 & $1.25 \times 10^{37}$ & & 2.0 & 50 & & \\
\hline & B & 7.0 & $1.25 \times 10^{38}$ & & 2.0 & 50 & & \\
\hline & $\mathrm{C}$ & 1.0 & $8.5 \times 10^{38}$ & & 2.0 & 50 & $0.9-1.0 \times 10^{39}$ & $\operatorname{ergs} s^{-1}$ \\
\hline
\end{tabular}

${ }^{1} W$ : Energy budget of high energy particles within individual clouds (see Fig. 2)

${ }^{2} \dot{W}$ : Particle injection luminosity of high energy particles into individual clouds

${ }^{3} W_{\text {SNR }}$ : Injected energy budget of high energy particles within progenitor SNR (see equation 11)

${ }^{4} \dot{W}_{\text {tot }}$ : Total injection luminosity of all three regions by PWN

Plausible scenarios are shown in bold

Matching scenarios have systematic variation of up to $56 \%$ in energy budget $W$ or luminosity $\dot{W}, 12 \%$ in the spectral index $\Gamma$ and $12 \%$ in the cutoff energy $E_{c}$ (see text and figure 8)

Table 3. Same as Table. 2 but parameters in a leptonic origin.

\begin{tabular}{|c|c|c|c|c|c|c|c|c|}
\hline \multirow{2}{*}{$\begin{array}{c}\text { Accelerator } \\
\text { PSR 1826-1334 or SNR }\end{array}$} & \multirow[b]{2}{*}{ Peak } & \multirow[b]{2}{*}{$n_{H}\left(\mathrm{~cm}^{-3}\right)$} & \multicolumn{4}{|c|}{ Leptonic } & \multirow[b]{2}{*}{$W_{\text {SNR }}$ or $\dot{W}_{\text {tot }}$} & \\
\hline & & & $W$ or $\dot{W}$ & & $\Gamma$ & $E_{C}(\mathrm{TeV})$ & & \multirow[b]{2}{*}{ erg } \\
\hline Impulsive (SNR) & $\mathrm{A}$ & 14 & $1.2 \times 10^{49}$ & erg & 2.0 & 10 & $7.3 \times 10^{50}$ & \\
\hline \multirow[t]{3}{*}{$t=21 \times 10^{3} \mathrm{yrs}$} & $\mathrm{B}$ & 79.8 & $9.0 \times 10^{48}$ & & 2.0 & 30 & $2.8 \times 10^{50}$ & \\
\hline & B & 7.0 & $8.0 \times 10^{48}$ & & 2.0 & 30 & $2.5 \times 10^{50}$ & \\
\hline & $\mathrm{C}$ & 1.0 & $7.0 \times 10^{48}$ & & 2.0 & 10 & $4.3 \times 10^{50}$ & \\
\hline Impulsive (SNR) & $\mathrm{A}$ & 14 & $1.4 \times 10^{49}$ & erg & 2.0 & 10 & $8.5 \times 10^{50}$ & erg \\
\hline \multirow[t]{3}{*}{$t=40 \times 10^{3} \mathrm{yrs}$} & $\mathrm{B}$ & 79.8 & $6.0 \times 10^{48}$ & & 1.0 & 50 & $1.9 \times 10^{50}$ & \\
\hline & B & 7.0 & $3.0 \times 10^{48}$ & & 1.5 & 50 & $9.4 \times 10^{49}$ & \\
\hline & $\mathrm{C}$ & 1.0 & $7.6 \times 10^{48}$ & & 2.0 & 50 & $4.6 \times 10^{50}$ & \\
\hline Continuous (PWN) & $\mathbf{A}$ & 14 & $1.5 \times 10^{37}$ & $\operatorname{erg~s}{ }^{-1}$ & 2.0 & 10 & & \\
\hline \multirow[t]{3}{*}{$t=21 \times 10^{3} \mathrm{yrs}$} & B & 79.8 & $1.5 \times 10^{37}$ & & 2.0 & 10 & & \\
\hline & B & 7.0 & $1.5 \times 10^{37}$ & & 2.0 & 10 & & \\
\hline & $\mathbf{C}$ & 1.0 & $1.0 \times 10^{37}$ & & 2.0 & 10 & $4.0 \times 10^{37}$ & $\operatorname{erg~s}^{-1}$ \\
\hline Continuous (PWN) & $\mathbf{A}$ & 14 & $1.0 \times 10^{37}$ & $\operatorname{ergs}^{-1}$ & 2.0 & 10 & & \\
\hline \multirow{3}{*}{$t=40 \times 10^{3} \mathrm{yrs}$} & B & 79.8 & $2.6 \times 10^{36}$ & & 1.7 & 10 & & \\
\hline & B & 7.0 & $1.6 \times 10^{36}$ & & 1.7 & 10 & & \\
\hline & $\mathbf{C}$ & 1.0 & $6.0 \times 10^{36}$ & & 2.0 & 10 & $1.8-1.9 \times 10^{37}$ & $\operatorname{erg~s}^{-1}$ \\
\hline
\end{tabular}

\subsubsection{HESS J1825-137 Progenitor}

A clear SNR rim can be seen in Fig. 4 connected to HESS J1825137. The projected area of the SNR, with radius of $140 \mathrm{pc}$, is assumed to be $\approx 64 \times 10^{3} \mathrm{pc}^{2}$. Note that the denser regions to the north of HESS J1825-137 shown in Fig 2 and 3 may dampen the northern expansion of the SNR associated with HESS J1825-137. This will affect the filling factor geometrically and in turn affect the inferred energy of the SNR as shown in Eq. 11. If no particles have escaped, it is expected that $10^{50} \mathrm{erg}$ of energy remains within the SNR. As the SNR is definitely well into its Sedov phase, some cosmic rays will have escaped the system, lowering the remaining energy within the SNR.
For an individual model to be successful, it must allow sufficient energetics within all three clouds simultaneously. If the particle energetics impacting one cloud is too large, the model will be rejected. For this reason when looking at Tables. 2 and 3, to determine if individual impulsive models were successful, the maximum energy budget/ injection luminosity will be compared to theoretical energetics.

\subsubsection{LS5039 Progenitor}

No clear SNR rim has been associated with LS 5039. If the age of LS 5039 is greater than $10^{5} \mathrm{yr}$ the SNR will have already dispersed into the The projected area of SNR will assume a minimum radius 
Table 4. Model parameters matching the observed emission of GeV-A, B and C for a hadronic scenario. The particle accelerators considered are the progenitor SNR associated with LS 5039 (impulsive) or the accretion of matter by the companion star onto LS 5039 (continuous). Example SED best fit is shown in Fig. 8. $W$ or $\dot{W}$ represents the energy budget. To see the regions used, refer to Fig. 3. The spectra of injected particles is represented by an exponential cutoff power law spectrum: $\frac{\mathrm{d} N}{\mathrm{~d} E} \propto E^{-\Gamma} \exp \left[E / E_{c}\right]$.

\begin{tabular}{|c|c|c|c|c|c|c|c|c|}
\hline $\begin{array}{c}\text { Accelerator } \\
\text { LS } 5039 \text { or SNR }\end{array}$ & Peak & $n_{H}\left(\mathrm{~cm}^{-3}\right)$ & $W$ or $\dot{W}$ & $\mathrm{H}$ & $\begin{array}{c}\text { Aronic } \\
\Gamma\end{array}$ & $E_{C}(\mathrm{TeV})$ & ${ }^{5} W_{\text {SNR }}$ or ${ }^{6} \dot{W}_{\text {total }}$ & \\
\hline Impulsive (SNR) & $\bar{A}$ & 7.0 & $8.0 \times 10^{49}$ & erg & 2.0 & 50 & $3.9 \times 10^{51}$ & erg \\
\hline \multirow[t]{2}{*}{$t=1 \times 10^{3} \mathrm{yrs}$} & B & 79.8 & $6.0 \times 10^{48}$ & & 2.0 & 50 & $1.5 \times 10^{50}$ & \\
\hline & $\mathrm{C}$ & 3.6 & $1.0 \times 10^{50}$ & & 2.0 & 50 & $4.9 \times 10^{51}$ & \\
\hline Impulsive (SNR) & A & 7.0 & $1.0 \times 10^{50}$ & erg & 2.0 & 50 & $4.9 \times 10^{51}$ & erg \\
\hline \multirow[t]{2}{*}{$t=1 \times 10^{5} \mathrm{yrs}$} & B & 79.8 & $8.0 \times 10^{48}$ & & 2.0 & 50 & $2.0 \times 10^{50}$ & \\
\hline & $\mathrm{C}$ & 3.6 & $1.5 \times 10^{50}$ & & 2.0 & 50 & $7.4 \times 10^{51}$ & \\
\hline Impulsive (SNR) & $\bar{A}$ & 7.0 & $3.0 \times 10^{51}$ & erg & 1.0 & 50 & $1.5 \times 10^{53}$ & erg \\
\hline \multirow[t]{2}{*}{$t=1 \times 10^{6} \mathrm{yrs}$} & B & 79.8 & $4.0 \times 10^{49}$ & & 1.5 & 50 & $1.0 \times 10^{51}$ & \\
\hline & $\mathrm{C}$ & 3.6 & $4.0 \times 10^{51}$ & & 1.0 & 50 & $2.0 \times 10^{53}$ & \\
\hline Continuous (accretion) & A & 7.0 & $2.3 \times 10^{39}$ & $\operatorname{erg~s}^{-1}$ & 2.0 & 50 & & \\
\hline \multirow[t]{2}{*}{$t=1 \times 10^{3} \mathrm{yrs}$} & B & 79.8 & $2.0 \times 10^{38}$ & & 2.0 & 50 & & \\
\hline & $\mathrm{C}$ & 3.6 & $4.5 \times 10^{39}$ & & 2.0 & 50 & $7.0 \times 10^{39}$ & $\operatorname{erg~s}^{-1}$ \\
\hline Continuous (accretion) & $\bar{A}$ & 7.0 & $2.8 \times 10^{37}$ & $\operatorname{erg~s}^{-1}$ & 2.0 & 50 & & \\
\hline \multirow[t]{2}{*}{$t=1 \times 10^{5} \mathrm{yrs}$} & B & 79.8 & $2.0 \times 10^{36}$ & & 2.0 & 50 & & \\
\hline & $\mathrm{C}$ & 3.6 & $4.0 \times 10^{37}$ & & 2.0 & 50 & $7.0 \times 10^{37}$ & $\operatorname{erg~s}^{-1}$ \\
\hline Continuous (accretion) & A & 7.0 & $2.5 \times 10^{36}$ & $\operatorname{erg~s}^{-1}$ & 2.0 & 50 & & \\
\hline \multirow[t]{2}{*}{$t=1 \times 10^{6} \mathrm{yrs}$} & B & 79.8 & $3.5 \times 10^{35}$ & & 2.0 & 50 & & \\
\hline & $\mathrm{C}$ & 3.6 & $4.0 \times 10^{36}$ & & 2.0 & 50 & $6.9 \times 10^{36}$ & $\operatorname{erg~s}^{-1}$ \\
\hline
\end{tabular}

\footnotetext{
${ }^{5} W_{\mathrm{SNR}}$ : Inferred energy budget of high energy particles inside progenitor SNR related to LS 5039

${ }^{6} \dot{W}_{\text {total }}$ : The total injection luminosity into all three clouds

Plausible scenarios are shown in bold

Matching scenarios have systematic variation of up to $56 \%$ in energy budget $W$ or luminosity $\dot{W}, 12 \%$ in the spectral index $\Gamma$ and $12 \%$ in the cutoff energy $E_{c}$ (see text and figure 8 )
}

Table 5. Same as Table. 4 but parameters in a leptonic scenario for gamma-ray emission.

\begin{tabular}{|c|c|c|c|c|c|c|c|c|}
\hline \multirow{2}{*}{$\begin{array}{c}\text { Accelerator } \\
\text { LS } 5039 \text { or SNR }\end{array}$} & \multicolumn{8}{|c|}{ Leptonic } \\
\hline & Peak & $n_{H}\left(\mathrm{~cm}^{-3}\right)$ & $W$ or $\dot{W}$ & & $\Gamma$ & $E_{C}(\mathrm{TeV})$ & $W_{\mathrm{SNR}}$ or $\dot{W}_{\mathrm{total}}$ & \\
\hline Impulsive (SNR) & $\bar{A}$ & 7.0 & $4.0 \times 10^{48}$ & erg & 2.0 & 10 & $2.0 \times 10^{50}$ & erg \\
\hline \multirow[t]{2}{*}{$t=1 \times 10^{3} \mathrm{yrs}$} & B & 79.8 & $2.5 \times 10^{48}$ & & 2.0 & 10 & $6.4 \times 10^{49}$ & \\
\hline & $\mathrm{C}$ & 3.6 & $3.0 \times 10^{48}$ & & 2.0 & 10 & $1.5 \times 10^{50}$ & \\
\hline Impulsive (SNR) & $\bar{A}$ & 7.0 & $5.0 \times 10^{48}$ & erg & 1.7 & 50 & $2.5 \times 10^{50}$ & erg \\
\hline \multirow[t]{2}{*}{$t=1 \times 10^{5} \mathrm{yrs}$} & B & 79.8 & $2.0 \times 10^{49}$ & & 1.0 & 10 & $5.1 \times 10^{50}$ & \\
\hline & $\mathrm{C}$ & 3.6 & $5.0 \times 10^{48}$ & & 2.0 & 10 & $2.5 \times 10^{50}$ & \\
\hline Impulsive (SNR) & $\bar{A}$ & 7.0 & $3.0 \times 10^{50}$ & erg & 1.7 & 100 & $1.5 \times 10^{52}$ & erg \\
\hline \multirow{2}{*}{$t=1 \times 10^{6} \mathrm{yrs}$} & B & 79.8 & & & & & & \\
\hline & $\mathrm{C}$ & 3.6 & $2.0 \times 10^{50}$ & & 2.0 & 10 & $9.9 \times 10^{51}$ & \\
\hline Continuous (accretion) & A & 7.0 & $1.0 \times 10^{38}$ & $\operatorname{erg~s}{ }^{-1}$ & 2.0 & 10 & & \\
\hline \multirow[t]{2}{*}{$t=1 \times 10^{3} \mathrm{yrs}$} & B & 79.8 & $8.5 \times 10^{37}$ & & 2.0 & 10 & & \\
\hline & $\mathrm{C}$ & 3.6 & $5.0 \times 10^{38}$ & & 2.0 & 10 & $6.9 \times 10^{38}$ & $\operatorname{erg~s}^{-1}$ \\
\hline Continuous (accretion) & $\mathrm{A}$ & 7.0 & $3.5 \times 10^{35}$ & $\operatorname{erg~s}^{-1}$ & 1.7 & 10 & & \\
\hline \multirow[t]{2}{*}{$t=1 \times 10^{5} \mathrm{yrs}$} & B & 79.8 & $9.0 \times 10^{35}$ & & 2.0 & 10 & & \\
\hline & $\mathrm{C}$ & 3.6 & $2.0 \times 10^{35}$ & & 1.5 & 10 & $1.5 \times 10^{36}$ & $\operatorname{erg~s}^{-1}$ \\
\hline Continuous (accretion) & $\overline{\mathbf{A}}$ & 7.0 & $2.0 \times 10^{35}$ & $\operatorname{erg~s}{ }^{-1}$ & 1.7 & 10 & & \\
\hline \multirow[t]{2}{*}{$t=1 \times 10^{6} \mathrm{yrs}$} & B & 79.8 & $7.5 \times 10^{35}$ & & 1.8 & 30 & & \\
\hline & C & 3.6 & $9.0 \times 10^{34}$ & & 1.5 & 10 & $1.0 \times 10^{36}$ & $\operatorname{erg~s}{ }^{-1}$ \\
\hline
\end{tabular}

of $\approx 80$ pc to completely encompass GeV-ABC as seen by FermiLAT. Equation 11 is then used to estimate the total energy of high energy particles remaining in the progenitor SNR of LS 5039. The assumed minimum radius of SNR will lead to an underestimation of the inferred energy of the SNR associated with LS 5039.

\section{DISCUSSION}

In this section we will discuss the results of the SED modelling and consider the possible accelerator scenarios.

\subsection{Accelerator related to HESS J1825-137}

Firstly we will examine the plausibility of an accelerator related to HESS J1825-137. The two sources of high energy particles are the progenitor SNR and PWN.

\subsubsection{A progenitor SNR (Impulsive)}

The progenitor SNR linked to HESS J1825-137 is an impulsive accelerator; releasing $\approx 10^{50}$ erg of cosmic rays (with electrons making up $\approx 10^{48} \mathrm{erg}$ ) into the surrounding environment. The SNR 

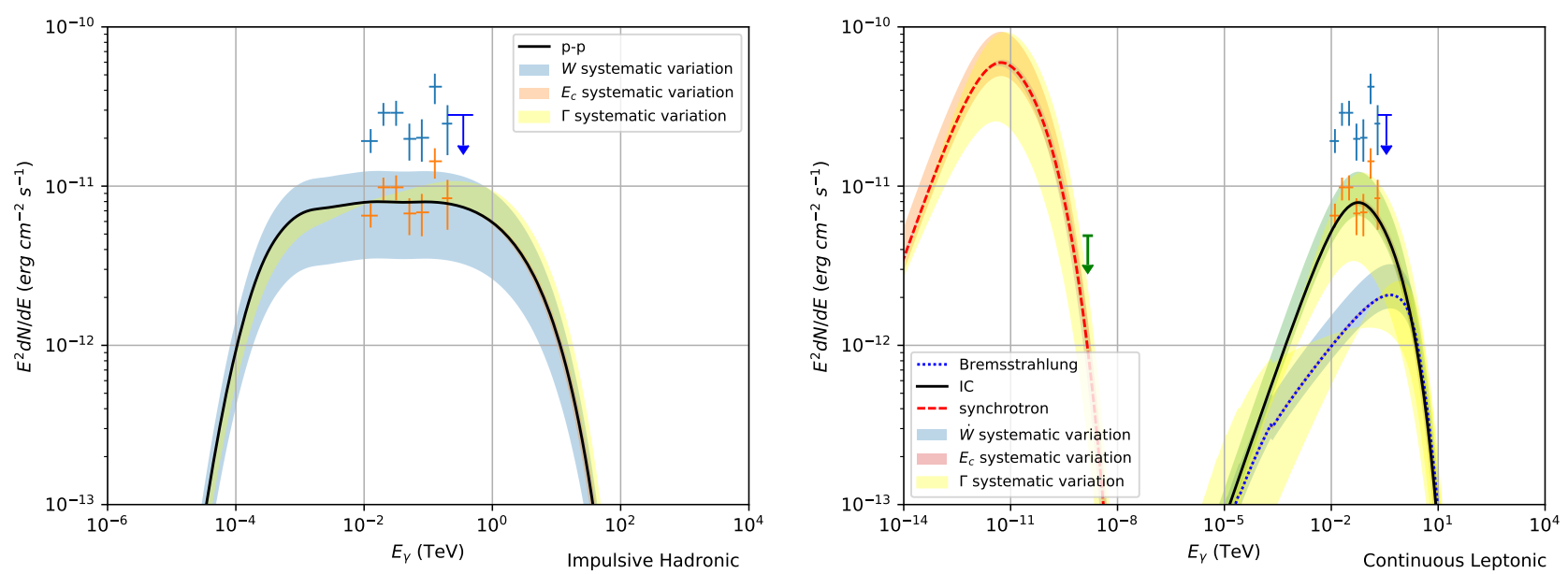

Figure 8. SED example for GeV-B with impulsive hadronic SED (left) and continuous leptonic SED (right). This assumes that HESS J1825-137 (at age $40 \mathrm{kyrs}$ ) is the source of acceleration. The upper data points represent the total SED as measured by Fermi-LAT. The lower data points is $34.0 \%$ of this spectra due to source being only GeV-B. The green arrow is the ROSAT X-ray upper limit towards GeV-ABC. The blue, pink and yellow shaded regions represent the systematic variation of energy budget $(W)$ or injection luminosity $(\dot{W})$, cutoff energy and spectral index respectively. See Tables 2 and 3 for input parameters.

expands and cosmic rays will escape from the system, decreasing the total energy of particles trapped inside the SNR. From spectral energy distribution modelling, the energy budget in regions $\mathrm{GeV}-\mathrm{A}$, $\mathrm{B}$ and $\mathrm{C}$ required to reproduce the SED of $\mathrm{GeV} \gamma$-rays was obtained. The total SNR cosmic ray energy budget, $W_{\mathrm{SNR}}$, is estimated by equation 11 .

To reproduce the SED of any of GeV-A, B or C requires the hadronic SNR energy budget to range between $5-730 \times 10^{50} \mathrm{erg}$, as shown in Table. 2. It is possible that HESS J1825-137 may be a possible hypernova candidate: supernova with kinetic energy greater than $10^{52} \mathrm{erg}$ (Nomoto et al. 2004). This is equivalent to a supernova releasing $10^{51}$ erg of cosmic rays. A plausible scenario requires for all three $\mathrm{GeV}$ regions to simultaneously explain the gamma-ray spectrum. For both ages ( $t=21$ and $40 \mathrm{kyr}$ ), only GeV-B has reasonable energetics assuming a higher ISM density; therefore a pure hadronic progenitor SNR scenario must be rejected unless a hypernova scenario is considered.

Hydrogen volume density is not constant across GeV-A, B and $\mathrm{C}$. Equation $\mathrm{C} 13$ shows that the spectra of gamma-rays from proton-proton interactions is proportional to the density of the target material. Assuming that the the high density cloud observed in the $15-30 \mathrm{~km} \mathrm{~s}^{-1}$ velocity range lies at the same distance as HESS J1825-137 then GeV-B should appear brighter in gamma-rays compared to $\mathrm{GeV}-\mathrm{A}$ and $\mathrm{C}$ assuming that the cosmic ray energy density over all three regions are constant. This is not the case, therefore the cosmic ray energy density in cloud A and C must be 7 and 80 times greater respectively than the energy density in cloud B. As discussed in section 4.1.2, particles escape the SNR at age $\sim 2 \mathrm{kyr}$ when it has a radius of $\sim 7 \mathrm{pc}$ and diffuse to $\mathrm{GeV}-\mathrm{ABC}$. By the time the particles have diffused the remaining distance to $\mathrm{GeV}$ $\mathrm{ABC}(\approx 130 \mathrm{pc})$ any local anisotropy at the GeV-ABC position will likely have been lost. Therefore an impulsive hadronic scenario cannot explain why GeV-A, B \& C have the same brightness.

A pure impulsive leptonic energy budget requires, at least, $10^{50} \mathrm{erg}$ of electrons within the SNR. Therefore a pure impulsive leptonic model of HESS J1825-137 being the accelerator of high energy particles resulting in the $\mathrm{GeV}$ gamma-radiation as observed by Fermi-LAT is rejected.

A leptonic-hadronic impulsive scenario requires leptonic in- teractions to produce $1 \%$ of the $\mathrm{GeV}$ gamma-rays as seen by FermiLAT to reduce the total SNR leptonic energy budget to $10^{48} \mathrm{erg}$. This leaves $99 \%$ of gamma-rays to be the result of hadronic interactions from SNR with energy budget of $5-720 \times 10^{50} \mathrm{erg}$. Therefore an impulsive scenario considering a combination of hadronic and leptonic interactions producing the observed $\mathrm{GeV}$ gamma-rays can be rejected.

\subsubsection{PWN (continuous)}

We will now examine the pulsar wind nebula as the source of high energy particles.

The spin down power of the pulsar powering PWN HESS J1825-137 is of order $10^{36} \mathrm{erg} \mathrm{s}^{-1}$. The spin down power of the pulsar is not constant over time; Aharonian et al. (2006) has suggested that the high gamma-ray luminosity may indicate that the spin-down power was far greater in the past.

From Table 2 a hadronic continuous scenario requires injection luminosities of $1.8 \times 10^{39} \mathrm{erg} \mathrm{s}^{-1}$ and $9.4 \times 10^{38} \mathrm{erg} \mathrm{s}^{-1}$ for ages $21 \mathrm{kyr}$ and $40 \mathrm{kyr}$ respectively. This far exceeds the spin down power of PSR J1826-1334, rejecting this scenario. Considering a leptonic continuous scenario for ages of $21 \mathrm{kyr}$ and $40 \mathrm{kyr}$, all three $\mathrm{GeV}$ regions require a total of $\approx 10^{37} \mathrm{erg} \mathrm{s}^{-1}$ in injection luminosity. If the spin down power of PSR J1826-1334 was greater in the past as suggested by Aharonian et al. (2006), GeV-ABC may be a reflection of an earlier epoch in the PWN history. The original spin-down power, $\dot{E}_{0}$ of the pulsar is linked to the present spin down power $E(t)$ through:

$\dot{E}(t)=\dot{E}_{0}\left(1+\frac{t}{\tau_{0}}\right)^{-\frac{n+1}{n-1}}$

where $n$ is the braking index of the pulsar and $\tau_{0}$ is the initial spindown timescale (Pacini \& Salvati 1973). The spin-down timescale can be determined from:

$\tau_{0}=\frac{P_{0}}{(n-1)\left|\dot{P}_{0}\right|}$

Taking the assumption from Principe et al. (2020) that $\dot{P}_{0}=$ $15 \mathrm{~ms}$ and assuming $\dot{P}=\dot{P_{0}}$ with a braking index of 3 , the original spin-down power of PSR J1826-1334, $\dot{E}_{0}$, was in the order of 
$10^{39} \mathrm{erg} \mathrm{s}^{-1}$. This exceeds the injection luminosity for a leptonic scenario with the PWN as the accelerator of high energy particles. Electrons injected into the PWN by the pulsar are transported by a combination of advection and diffusion. At the edge of the PWN, it can be assumed that the electrons escape isotropically. Consequently the GeV gamma-ray emission towards $\mathrm{GeV}-\mathrm{ABC}$ is expected to follow the photon fields through IC interactions. As the CMB photon field is constant, only the IR photon field would affect the morphology of gamma-ray emission. As seen in Fig. 10 the peaks in the $\mathrm{GeV}$ gamma-ray emission do not correspond to the IR field. Under this scenario, a preferential direction would be required for the advection/diffusion of electrons from the PWN.

Fig. 6 shows diffusive particle transport of electrons travelling a distance of $140 \mathrm{pc}$ in ambient density of $n=1 \mathrm{~cm}^{-3}$ versus the cooling of synchrotron and IC processes. This is equivalent to the distance that electrons travel after being emitted by the pulsar to reach GeV-B. the vertical lines represent the equivalent minimum and maximum electron energy seen by Fermi-LAT and H.E.S.S.respectively. Fast diffusion $(\chi=1.0)$ is required for electrons in this energy range to reach GeV-B within the age of PWN.

The High Altitude Water Cherenkov Observatory (HAWC) has observed $\gamma$-rays greater than $100 \mathrm{TeV}$ (HAWC Collaboration et al. 2019 ) suggesting that $E_{\mathrm{e}, \max }$ is greater than shown in figure 6 . The maximum electron electron able to reach GeV-B is determined by the intersection of diffusion time and the cooling time, i.e. where all electrons have lost their energy through leptonic interactions. On the other hand the minimum electron energy is represented by the intersection of diffusion time and the age of the pulsar. It can be concluded that for slow diffusion $(\chi=0.01)$ no electrons are able to reach GeV-B; while for fast diffusion, electrons greater than $\approx 10 \mathrm{TeV}$ can travel to GeV-B in time. This is reaching the cutoff energy required to reproduce the spectral energy distribution of leptonic process as seen in Table 3.

A more powerful pulsar can convert more of its spin down power into electron energy, allowing a greater proportion of higher energy electrons. This, in turn, allows more electrons to reach GeV$\mathrm{ABC}$ in time to emit $\mathrm{GeV}$ radiation. Therefore unless advection or fast diffusion is considered or the PWN is powerful, electrons are unable to reach GeV-ABC from PSR J1826-1334 without significant energy losses.

\subsection{LS 5039 as a particle accelerator}

We will now discuss the possibility of LS 5039 as the accelerator for high energy particles resulting in gamma-rays observed towards $\mathrm{GeV}-\mathrm{ABC}$.

\subsubsection{Progenitor SNR (Impulsive)}

From Table 4, if GeV-ABC is the result of hadronic interactions from an impulsive progenitor SNR, no age of LS 5039 can simultaneously explain the $\mathrm{GeV}$ emission as total energy budgets exceed $10^{50} \mathrm{erg}$. Due to the denser cloud towards GeV-B as seen in Fig. 2 for all three clouds to be explained by the same source of high energy particles, the cosmic ray density must be approximately a factor of 10 larger in GeV-B than GeV-A and GeV-C. It can be concluded that an impulsive hadronic source of cosmic rays from LS 5039 cannot simultaneously explain the $\mathrm{GeV}$ regions observed by Araya et al. (2019). Similarly an impulsive leptonic source for any age of LS 5039 cannot explain any of the $\mathrm{GeV}$ emission from $\mathrm{GeV}-\mathrm{ABC}$ due to energy budgets exceeding $10^{49} \mathrm{erg}$ as shown in Table. 5 .

\subsubsection{Accretion powered (continuous)}

Microquasars such as LS 5039 are considered to be candidates for particle acceleration up to gamma-ray energies (Aharonian et al. 2005). The average accretion luminosity of LS 5039 is $8.0 \times 10^{35} \mathrm{erg} \mathrm{s}^{-1}$ (Casares et al. 2005). Approximately one third of accreted energy is radiated in the relativistic jet (Casares et al. 2005). The remaining $5.3 \times 10^{35} \mathrm{erg}$ is assumed to be channelled into $\mathrm{GeV}$ $\mathrm{ABC}$ through a jet. It is unknown whether this jet is hadronic or leptonic in origin. This jet is a continuous source of particles into the region towards $\mathrm{GeV}-\mathrm{ABC}$. While the jet generally points in the direction of GeV-B (see Fig 4), the precession of the jet may allow electrons to be channelled into GeV-A and GeV-C. Particles may also diffuse from the jet escaping into the necessary regions.

A hadronic scenario requires a total injection luminosity into $\mathrm{GeV}-\mathrm{ABC}$ of $10^{39}-10^{36} \mathrm{erg} \mathrm{s}^{-1}$ for an age range of $10^{3}-10^{6} \mathrm{yrs}$. All ages require total injection luminosity greater than the accretion luminosity can provide; rejecting a hadronic accretion powered scenario.

On the other hand a leptonic scenario requires a total injection luminosity into $\mathrm{GeV}-\mathrm{ABC}$ of $10^{38}-10^{36} \mathrm{erg} \mathrm{s}^{-1}$ for an age range of $10^{3}-10^{6} \mathrm{yrs}$. The younger ages of $10^{3} \mathrm{yrs}$ and $10^{5} \mathrm{yrs}$ can be rejected. All scenarios can vary systematically up to $56 \%$ in injection luminosity, an age of $10^{6}$ yrs can provide the energetics required to reproduce the gamma-rays as seen by Fermi-LAT. But this age is greater than the age of $\approx 10^{5}$ yrs predicted by Moldón et al. (2012). Therefore a leptonic scenario with a continuous jet powered by the accretion onto compact object in LS 5039 can be rejected.

Using the calculated hydrogen densities towards the regions of interest in LS 5039 in the $40-60 \mathrm{~km} \mathrm{~s}^{-1}$ range rather than the $15-30 \mathrm{~km} \mathrm{~s}^{-1}$ range will not alter the results due to values being within a factor of 10 of each other.

In summary, it is unlikely that LS 5039 is the source of the new region of $\mathrm{GeV}$ gamma-ray emission.

\subsection{Combination of LS 5039 and HESS J1825-137}

The new region of GeV gamma-rays may be a line of sight combination of HESS J1825-137 and LS 5039. As discussed in section 5.1 and 5.2 a hadronic scenario requires cosmic ray energy density to be ten times higher in GeV-A and GeV-C compared to GeV-B assuming the dense gas observed in the velocity range $15-30 \mathrm{~km} / \mathrm{s}$ in Fig. 2 lies at the same distance as HESS J1825-137. Note in the case of HESS J1825-137, it assumes the dense gas observed in $15-30 \mathrm{~km} \mathrm{~s}^{-1}$ range in Fig. 2 lies at the same distance as HESS J1825-137. If the GeV gamma-ray emission from GeV-A and $\mathrm{GeV}-\mathrm{C}$ is unrelated to emission from GeV-B, this issue will be negated.

As seen in Fig. 4 the region around GeV-ABC contains several astrophysical environments; a $\mathrm{H} \alpha$ region believed to be associated with the SNR linked to HESS J1825-137 and a relativistic jet from LS 5039. Even though HESS J1825-137 and LS 5039 are at different distances ( $3.9 \mathrm{kpc}$ and $2.5 \mathrm{kpc}$ respectively), the combination of these two processes may explain the spectra observed by FermiLAT.

Peaks GeV-B and GeV-C have similar spectral indices, $\Gamma=$ $1.7 \pm 0.4$ and $\Gamma=1.78 \pm 0.25$ respectively, indicating a common origin scenario, whilst GeV-A has a marginally harder spectra with $\Gamma=1.43 \pm 0.23$. GeV-A is positioned the closest to both HESS J1825-137 and LS 5039. As shown by equation 3 and 4, high energy particles are able to travel further distances than lower en- 


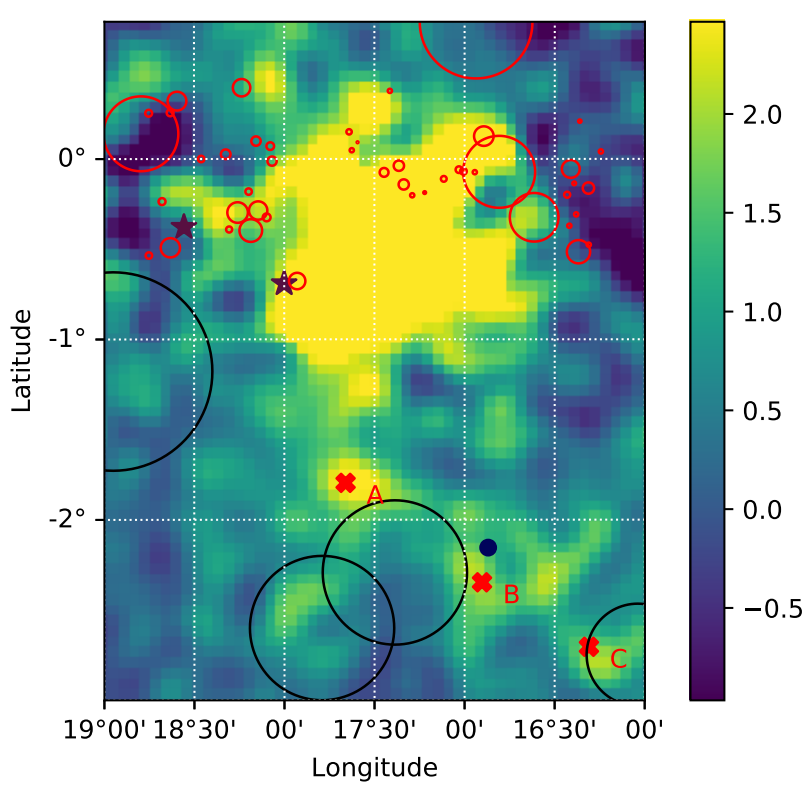

Figure 9. Fermi-LAT count map above $10 \mathrm{GeV}$ (Araya et al. 2019) towards HESS J1825-137 is shown with alternative accelerators for high energy particles towards HESS J1825-137 and GeV-ABC. Red circles describe HII regions where star formation may occur as given by the WISE catalogue (Anderson 2014). Black circles show the location of other SNR towards the region of interest as described in section 5.4. Dark purple stars represent pulsars PSR J1826-1334 and PSR J1826-1256 (Manchester et al. 2005). The water maser, G016.8689-02.1552, can be seen as a dark blue dot nearby GeV-B (Urquhart et al. 2011)

ergy particles in the same time. Clouds closer to the source of high energy particles are expected to have a softer spectrum than clouds lying further from the source for both continuous and impulsive sources (Aharonian \& Atoyan 1996). This is the opposite to what is observed in GeV-ABC.

\subsection{Particle Accelerators unrelated to HESS J1825-137 and LS 5039}

Towards GeV-ABC, there are four known supernova remnants; these are SNR G017.4-02.3, SNR G018.9-01.1, SNR G016.2-02.7 and SNR G017.8-02.6; see figure 9. From equation 5c, the cooling time of electrons resulting in synchrotron emission is proportional to the energy; as the supernova remnant ages, higher energy electrons escape from the system or lose their energy decreasing the emitted photon energy. Therefore as a supernova remnant ages, the amount of X-ray emission detected decreases. Three of the four supernova remnants have no current X-ray detection, indicating that these SNRs are old (at least in the later stages of the Sedov-Taylor phase). They are therefore unlikely to be a source of high energy particle acceleration, resulting in the production of $\mathrm{GeV}$ gamma-rays. The remaining supernova remnant, SNR G18.9-1.1, has a partial X-ray shell (Harrus et al. 2004). Based on radio measurements by Harrus et al. (2004) it is located 2 or $15.1 \mathrm{kpc}$ away. More recent research indicates a distance of $2.1 \pm 0.4 \mathrm{kpc}$ and age of $3700 \mathrm{yr}$ (Ranasinghe et al. 2019). As mentioned by Araya et al. (2019), if GeV-ABC is the result of a combination of SNRs, Fermi-LAT images will show distinct sources above $10 \mathrm{GeV}$ with the given Fermi-LAT resolution. Star forming regions have also been suggested as an accelerator of cosmic rays. See Fig. 9 to see location of star forming

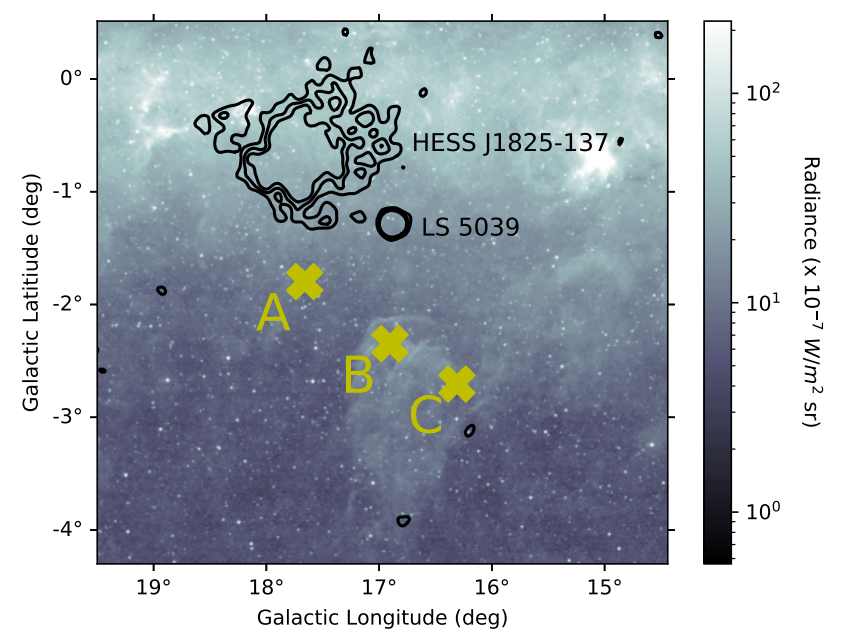

Figure 10. Infrared emission in the $8.26 \mu \mathrm{m}$ band towards $\mathrm{GeV}-\mathrm{ABC}$ (Egan et al. 2003). Overlayed are the HESS significance contours towards HESS J1825-137 at $1 \sigma, 2 \sigma$ and $3 \sigma$ (H. E. S. S. Collaboration et al. 2018).

regions, SNRs and pulsars towards HESS J1825-137 and GeV-ABC. The presence of water maser G016.8689-02.1552, as shown in Fig. 9, highly suggests star formation towards this region (Urquhart et al. 2011). This is supported by data from the MSX satellite; data reveals infra-red emission towards GeV-B and GeV-C in the $8.26 \mu \mathrm{m}$ band (see Fig. 10).

\section{CONCLUSIONS}

This study presented spectral models of a region of GeV gamma-ray emission to the south of HESS J1825-137 revealed by Fermi-LAT. Different accelerators were proposed to be an origin for high energy particles that created this new region of gamma-rays; the PWN (continuous) and SNR (impulsive) associated with HESS J1825137, and the binary system and microquasar LS 5039 (continuous) as well as the associated progenitor SNR (impulsive). We found that the progenitor SNR related to HESS J1825-137 is unlikely to be the sole source of high energy particles due to the energetics needed to replicate the SED is greater than what the system can provide. For example an impulsive SNR releases approximately $10^{50} \mathrm{erg}$ of cosmic rays (with $1 \%$ of energy channeled into electrons), whereas the SED model of the progenitor SNR of HESS J1825-137 is required to provide either $10^{52} \mathrm{erg}$ of protons or $10^{50} \mathrm{erg}$ of electrons to replicate the spectral energy distribution. A continuous acceleration scenario from the PWN (powered by the pulsar) into GeV-ABC requires particle injection luminosity to be of order $10^{39} \mathrm{erg} \mathrm{s}^{-1}$ and $10^{37} \mathrm{erg} \mathrm{s}^{-1}$ for hadronic and leptonic particles respectively. GeV$\mathrm{ABC}$ may be a reflection of an earlier epoch in the PWN history, where the pulsar was more powerful. Therefore the PWN may be a possible accelerator for high energy electrons resulting in this new region of gamma-ray emission, assuming fast diffusion perhaps including advection towards this region. Moreover, it is unlikely that leptonic inverse-Compton emission into this region will produce the localised features such as GeV-ABC. LS 5039 at any age cannot solely explain the $\mathrm{GeV}$ emission from $\mathrm{GeV}-\mathrm{ABC}$ with required injection luminosity > $10^{36} \mathrm{erg} \mathrm{s}^{-1}$ compared to the $10^{35} \mathrm{erg} \mathrm{s}^{-1}$ accretion luminosity of LS 5039 (Casares et al. 2005). However a combination of emission from both HESS J1825-137 and LS 5039 could be the cause of the gamma rays. 


\section{ACKNOWLEDGEMENTS}

This research has made use of the NASA's Astrophysics Data System and the SIMBAD database, operated at CDS, Strasbourg, France. T.C. acknowledges support through the provision of Australian Government Research Training Program Scholarship.

\section{DATA AVAILABILITY}

No new data were generated or analysed in support of this research.

\section{REFERENCES}

Aharonian F. A., Atoyan A. M., 1996, A\&A, 309, 917

Aharonian F., et al., 2005, Science, 309, 746

Aharonian F., et al., 2006, A\&A, 460, 365

Anderson L. D., 2014, in American Astronomical Society Meeting Abstracts \#223. p. 312.01

Araya M., Mitchell A. M. W., Parsons R. D., 2019, Mon. Not. Roy. Astron. Soc., 485, 1001

Berezinskii V. S., Bulanov S. V., Dogiel V. A., Ptuskin V. S., 1990, Astrophysics of cosmic rays

Blumenthal G. R., Gould R. J., 1970, Reviews of Modern Physics, 42, 237 Bosch-Ramon V., Paredes J. M., Ribó M., Miller J. M., Reig P., Martí J., 2005, ApJ, 628, 388

Brand J., Blitz L., 1993, A\&A, 275, 67

Casares J., Ribó M., Ribas I., Paredes J. M., Martí J., Herrero A., 2005, MNRAS, 364, 899

Crutcher R. M., 1999, The Astrophysical Journal, 520, 706

Crutcher R. M., Wandelt B., Heiles C., Falgarone E., Troland T. H., 2010, ApJ, 725, 466

Draine B. T., 2011, Physics of the Interstellar and Intergalactic Medium

Egan M. P., Price S. D., Kraemer K. E., 2003, in American Astronomical Society Meeting Abstracts. p. 57.08

Finkbeiner D. P., 2003, ApJS, 146, 407

Gabici S., Aharonian F. A., Blasi P., 2007, Ap\&SS, 309, 365

Gabici S., Aharonian F. A., Casanova S., 2009, MNRAS, 396, 1629

H. E. S. S. Collaboration et al., 2018, A\&A, 612, A1

H. E. S. S. Collaboration et al., 2019, A\&A, 621, A116

HAWC Collaboration et al., 2019, arXiv e-prints, p. arXiv:1909.08609

Harrus I. M., Slane P. O., Hughes J. P., Plucinsky P. P., 2004, ApJ, 603, 152

Hinton J. A., Hofmann W., 2009, ARA\&A, 47, 523

Liu R.-Y., Yan H., 2020, MNRAS, 494, 2618

Manchester R. N., Hobbs G. B., Teoh A., Hobbs M., 2005, AJ, 129, 1993

Manolakou K., Horns D., Kirk J. G., 2007, aap, 474, 689

Marcote B., Ribó M., Paredes J. M., Ishwara-Chandra C. H., 2015, MNRAS, 451, 59

Marti J., Paredes J. M., Ribo M., 1998, A\&A, 338, L71

McClure-Griffiths N. M., et al., 2009, ApJS, 181, 398

Mizuno A., Fukui Y., 2004, Physical properties of molecular clouds as revealed by NANTEN CO survey: from the galactic center to the galactic warp. p. 59

Moderski R., Sikora M., Coppi P. S., Aharonian F., 2005, MNRAS, 363, 954

Moldón J., Ribó M., Paredes J. M., Brisken W., Dhawan V., Kramer M., Lyne A. G., Stappers B. W., 2012, A\&A, 543, A26

Motch C., Haberl F., Dennerl K., Pakull M., Janot-Pacheco E., 1997, A\&A, 323,853

Nomoto K., Maeda K., Mazzali P. A., Umeda H., Deng J., Iwamoto K., 2004 Hypernovae and Other Black-Hole-Forming Supernovae. pp 277-325, doi:10.1007/978-0-306-48599-2 10

Pacini F., Salvati M., 1973, ApJ, 186, 249

Paredes J. M., Ribó M., Ros E., Martí J., Massi M., 2002, A\&A, 393, L99

Principe G., Mitchell A., Hinton J., Parson D., Caroff S., Hahn J., Funk S., 2019, in 36th International Cosmic Ray Conference (ICRC2019). p. 595 (arXiv: 1909.11367)
Principe G., Mitchell A. M. W., Caroff S., Hinton J. A., Parsons R. D., Funk S., 2020, A\&A, 640, A76

Ptuskin V. S., Zirakashvili V. N., 2005, A\&A, 429, 755

Ranasinghe S., Leahy D., Tian W. W., 2019, arXiv e-prints, p. arXiv: 1910.05407

Reynolds S. P., 2008, ARA\&A, 46, 89

Sabol E. J., Snowden S. L., 2019, sxrbg: ROSAT X-Ray Background Tool (ascl:1904.001)

Strong A. W., Moskalenko I. V., Reimer O., Digel S., Diehl R., 2004, A\&A, 422, L47

Stupar M., Parker Q. A., Filipović M. D., 2008, Monthly Notices of the Royal Astronomical Society, 390, 1037

Taylor J. H., Cordes J. M., 1993, ApJ, 411, 674

Urquhart J. S., et al., 2011, MNRAS, 418, 1689

Voisin F., Rowell G., Burton M. G., Walsh A., Fukui Y., Aharonian F., 2016, MNRAS, 458, 2813

de Jager O. C., Djannati-Ataï A., 2009, Implications of HESS Observations of Pulsar Wind Nebulae. Springer, Berlin, Heidelberg, p. 451, doi:10.1007/978-3-540-76965-1_17

van der Swaluw E., Wu Y., 2001, ApJ, 555, L49

\section{APPENDIX A: ISM PARAMETERS}

Table A1: Calculated molecular parameters for HESS J1825-137 and the new $\mathrm{GeV}$ emission regions $\mathrm{GeV}-\mathrm{A}, \mathrm{GeV}-\mathrm{B}, \mathrm{GeV}-\mathrm{C}$ as shown in Fig. 2. $M_{H}$ and $n_{H}$ describes the average mass and density respectively over the new $\mathrm{GeV}$ regions.

\begin{tabular}{cccc}
\hline $15-30 \mathrm{~km} \mathrm{~s}^{-1}$ & Region & $M_{H}\left(M_{\odot}\right)$ & $n_{H}\left(\mathrm{~cm}^{-3}\right)$ \\
\hline & HESS J1825-137 & $1.14 \times 10^{5}$ & 39 \\
& GeV-A & $3.67 \times 10^{3}$ & 5 \\
& GeV-B & $1.36 \times 10^{5}$ & 79 \\
& GeV-C & $1.53 \times 10^{3}$ & 2 \\
\hline $40-60 \mathrm{~km} \mathrm{~s}^{-1}$ & Region & $M_{H}\left(M_{\odot}\right)$ & $n_{H}\left(\mathrm{~cm}^{-3}\right)$ \\
\hline & HESS J1825-137 & $5.18 \times 10^{5}$ & 176 \\
& GeV-A & $8.49 \times 10^{3}$ & 13 \\
GeV-B & $1.21 \times 10^{4}$ & 7 \\
GeV-C & \multicolumn{2}{c}{ No ISM values } \\
\hline
\end{tabular}

Table A2: Calculated HI densities for HESS J1825-137 and the new $\mathrm{GeV}$ emission regions $\mathrm{GeV}-\mathrm{A}, \mathrm{GeV}-\mathrm{B}$ and $\mathrm{GeV}-\mathrm{C}$.

\begin{tabular}{cccc}
\hline $15-30 \mathrm{~km} \mathrm{~s}^{-1}$ & Object & $M_{H}\left(M_{\odot}\right)$ & $n_{H}\left(\mathrm{~cm}^{-3}\right)$ \\
\hline & HESS J1825-137 & $3.53 \times 10^{3}$ & 1.2 \\
& GeV-A & $8.91 \times 10^{2}$ & 1.4 \\
GeV-B & $1.51 \times 10^{3}$ & 0.9 \\
& GeV-C & $7.70 \times 10^{2}$ & 1.2 \\
\hline $40-60 \mathrm{~km} \mathrm{~s}^{-1}$ & Object & $M_{H}\left(M_{\odot}\right)$ & $n_{H}\left(\mathrm{~cm}^{-3}\right)$ \\
\hline & HESS J1825-137 & $4.29 \times 10^{3}$ & 1.5 \\
& GeV-A & $4.36 \times 10^{2}$ & 0.7 \\
& GeV-B & $5.06 \times 10^{2}$ & 0.3 \\
& GeV-C & $3.11 \times 10^{2}$ & 0.5 \\
\hline
\end{tabular}

Table A3: Calculated H $\alpha$ densities for HESS J1825-137 and new $\mathrm{GeV}$ emission regions.

\begin{tabular}{ccc}
\hline Object & Method A $\left(\mathrm{cm}^{-3}\right)$ & Method B $\left(\mathrm{cm}^{-3}\right)$ \\
\hline HESS J1825-137 & $8.88 \times 10^{-6}$ & $4.12 \times 10^{-6}$ \\
GeV-A & $1.12 \times 10^{-6}$ & $2.40 \times 10^{-6}$ \\
GeV-B & $6.49 \times 10^{-6}$ & $5.13 \times 10^{-6}$ \\
GeV-C & $2.45 \times 10^{-6}$ & $5.23 \times 10^{-6}$ \\
\hline
\end{tabular}




\section{APPENDIX B: H $\alpha$ DENSITY CALCULATION METHOD}

\section{Method A}

Method A assumes that the density of photons in the region of interest is approximately equal to the density of ionised gas $n \approx u_{\mathrm{ph}}$. This assumes that atoms are not being re-excited by an external source. Considering a spherical shell located at distance $d$ from the source with thickness $\mathrm{d} \ell$; the volume of the shell is given by $\mathrm{d} V=4 \pi d^{2} \times \mathrm{d} \ell$. Photons emitted by the source travel at the speed of light, therefore $\mathrm{d} \ell=c \mathrm{~d} t$. The number of photons emitted by the source in time $\mathrm{d} t$ is related to the luminosity $L$ through $\mathrm{d} N=L \mathrm{~d} t$. Using the original approximation, the density of ionised hydrogen in a region of interest:

$n \approx u_{\mathrm{ph}}=\frac{\mathrm{d} N}{\mathrm{~d} V}=\frac{L}{4 \pi d^{2} c}$

Let the region of interest have solid angle $\Omega$ and lying at distance $d$. The luminosity of the region is given by:

$L[$ photon $/ \mathrm{s}]=\frac{d^{2}}{10^{-10}} \Omega I$

where $I$ is the measured $\mathrm{H} \alpha$ intensity in Rayleigh units.

\section{Method B}

Method B considers basic radiation transfer. The density of atoms in the $i$ th excited state emit photons at frequency $v$ through spontaneous emission is related to the emission coefficient by:

$n_{i}=\frac{j_{\nu} \Omega_{\text {Earth }}}{E_{v} A \phi(v)}$

where A is the Einstein coefficient, $\Omega_{\text {earth }}$ is the solid angle of Earth projected at source lying at distance $d$ and $\phi(v)$ is the spectral line shape normalised by:

$\int \phi(v)=1$

Assuming that hydrogen atoms in the $n=3$ state emit mainly $\mathrm{H} \alpha$ light; $\phi=0$ in all frequencies except when $v=v_{H \alpha}$. The photon radiance $L_{\text {rad }}$ is related to the intensity $I$ in Rayleigh's through:

$L_{\text {rad }}\left[\right.$ photons $\left./ \mathrm{m}^{2} / \mathrm{s} / \mathrm{sr}\right]=\frac{L}{4 \pi d^{2}}$

The photon intensity can be found utilising $I_{v}=L \frac{E_{v}}{v}=h L$ where $\mathrm{h}$ is Planck's constant. Let $s$ be the thickness of gas in the line of sight and assuming the emission coefficient is constant, the emission coefficient and intensity are related by:

$j_{v}=\frac{I_{\nu}}{S}$

This can be used in combination with equation B3 to obtain the photon density.

\section{APPENDIX C: SED MODEL}

The SED modelling code includes various astrophysical processes; included proton-proton interactions: $\mathrm{p}+\mathrm{p} \rightarrow \pi^{+}+\pi^{-}+\pi^{0}$

$\pi^{0} \stackrel{\text { decay }}{\longrightarrow} \gamma+\gamma$

Inverse Compton interactions:

$\mathrm{e}^{-*}+\gamma^{*} \rightarrow \mathrm{e}^{-}+\gamma$

Bremsstrahlung interactions with a nucleus with proton number $Z$ :

$\mathrm{e}^{-*}+Z \rightarrow \gamma+\mathrm{e}^{-}+Z$

and synchrotron interactions:

$\mathrm{e}^{-*}+\vec{B} \rightarrow \mathrm{e}^{-}$

The evolution of the cosmic ray energy distribution with Lorentz factor $\gamma$ at time $t$ is given by:

$\frac{\partial n(\gamma, t)}{\partial t}=\frac{\partial}{\partial \gamma}[\dot{\gamma}(\gamma) n(\gamma, t)]+S(\gamma, t)$

where $S(\gamma, t)$ is the source term, $\dot{\gamma}(\gamma)$ represents the energy loss rate of a particle with Lorentz factor $\gamma$. The analytical solution of equation $\mathrm{C6}$ is:

$n(\gamma, t)=\frac{1}{\dot{\gamma}} \int_{\gamma}^{\gamma_{0}} S\left(\gamma^{\prime \prime}, t-\tau\left(\gamma^{\prime \prime}\right)\right) \mathrm{d} \gamma^{\prime \prime}+\frac{\dot{\gamma}_{0}}{\gamma} n\left(\gamma_{0}, 0\right)$

where $\tau$ is a variable describing the time for a cosmic ray with initial Lorentz factor $\gamma^{\prime}$ to evolve to factor $\gamma$ :

$\tau\left(\gamma^{\prime}, \gamma\right)=\int_{\gamma}^{\gamma^{\prime}} \frac{\mathrm{d} \gamma^{\prime \prime}}{\dot{\gamma}\left(\gamma^{\prime \prime}\right)}$

and $\gamma_{0}$ is the initial Lorentz factor. The code solves equation C7 considering hadronic and leptonic interactions and then extracts the spectral energy distribution. The model allows the user to choose whether the case is leptonic, hadronic or a mixture. Similarly the user can choose if the model is continuous (constant cosmic ray input, eg a PWN) or impulsive (releases all the cosmic rays at once, eg a SNR). Other parameters such as the age and distance from the source, density and magnetic field of background material, the total energy and spectral distribution of cosmic rays and background photon field energy distribution can be changed depending on the source.

To find the SED at time $t$, for each lorentz factor $\gamma$ the lorentz factor at earlier time, $\gamma_{0}$ is derived. In the case of an impulsive source $\gamma_{0}$ is simply $\gamma$ at $t=0$. The total cooling rate is given by Manolakou et al. (2007):

$\dot{\gamma}(\gamma)=\cdot\left\{\begin{array}{l}b_{s} \gamma^{2}+b_{c}(3 \ln \gamma+18.8)+5.3 b_{b^{+}} \\ \sum_{t=i} b_{\mathrm{IC}}^{i} \gamma^{2} F_{\mathrm{KN}}^{i}(\gamma), \\ \frac{1}{n_{H} c \sigma_{\mathrm{pp}(\gamma)}},\end{array}\right.$ for leptonic cases for hadronic cases

where: 


\section{T. Collins et al.}

- $b_{s}=1.292 \times 10^{-15}\left(B / 10^{3} \mu \mathrm{g}\right)^{2} \mathrm{~s}^{-1}$ is a synchrotron loss constant.

- $b_{c}=1.491 \times 10^{-14}\left(n_{H} / 1 \mathrm{~cm}^{-3}\right)$ is the Coulomb loss constant.

- $b_{b}=1.37 \times 10^{-16}\left(n_{H} / 1 \mathrm{~cm}^{-3}\right) \mathrm{s}^{-1}$ is the Bremsstrahlung loss constant.

- $b_{\mathrm{IC}}=5.204 \times 10^{-20}\left(u_{0}^{i} / \mathrm{eV}\right) \mathrm{s}^{-1}$ is a IC loss constant with the energy density of photons given by $u_{0}$.

- $\sum_{t=i}$ sums over all radiation fields contributing to the Inverse compton gamma-ray flux.

- $n_{H}$ is the density of the ambient hydrogen gas.

- $\sigma_{\mathrm{pp}(\gamma)}$ is the cross section for proton-proton interactions.

To obtain $\gamma_{0}$, the following two steps are repeated until $t=t_{\text {age }}$ :

(i) derive $\Delta t=\mathrm{d} \gamma / \dot{\gamma}(\gamma)$

(ii) Increment $\gamma$ by $\mathrm{d} \gamma$

with automatic adjustion of the $\mathrm{d} \gamma$ step. Another parameter in the code is "escape". If this parameter is activated, once a particle escapes the system, it is no longer considered. The final synchrotron flux is given by:

$P(v)=\frac{\sqrt{3} e^{3} B}{m c^{2}} \frac{v}{v_{c}} \int_{\frac{v}{v_{c}}}^{\infty} K_{\frac{5}{3}}(x) \mathrm{d} x$

where $e$ and $m$ are the charge and mass of an electron respectively, $v$ is the frequency of the gamma-ray, $v_{c}$ is the critical frequency of the emission, and $K_{\frac{5}{3}}$ is the modified Bessel Function. The final Inverse Compton flux radiated by a single electron with energy $\epsilon$ is given by:

$\frac{\mathrm{d} N}{\mathrm{~d} E_{\gamma}}=\frac{3}{4} \sigma_{T} c \int \frac{n(\epsilon) \mathrm{d} \epsilon}{\epsilon} F_{\mathrm{KN}}\left(E_{e}, E_{\gamma}, \epsilon\right)$

where $\sigma_{T}$ is the Thompson cross section and $F_{\mathrm{KN}}$ is the KleinNishina cross section. The final Bremsstrahlung Flux is given by:

$\frac{\mathrm{d} N}{\mathrm{~d} E_{\gamma}}=n c \int \mathrm{d} \sigma\left(E_{e}, E_{\gamma}, Z\right) \mathrm{d} E_{e}$

where $Z$ is the atomic number of the target material and $\mathrm{d} \sigma$ is defined in Blumenthal \& Gould (1970). Finally proton-proton interactions produce a flux of:

$\frac{\mathrm{d} N}{\mathrm{~d} E_{\gamma}}=n c \int_{E_{p}=E_{\gamma}}^{\infty} A_{\max }\left(T_{p}\right) F\left(E_{\gamma}, T_{p}\right) \mathrm{d} E_{p}$

where $n$ is the density of protons, $A_{\max }\left(T_{p}\right)$ is the pion production cross section, $T_{p}$ is the kinetic energy of the proton and $F\left(E_{\gamma}, T_{p}\right)$ is the spectra of gamma rays emitted for a single proton of energy $E_{p}$.

\section{APPENDIX D: OTHER SPECTRAL ENERGY DISTRIBUTION PLOTS}

This paper has been typeset from a $\mathrm{T}_{\mathrm{E}} \mathrm{X} / \mathrm{L} \mathrm{AT} \mathrm{E} \mathrm{X}$ file prepared by the author. 

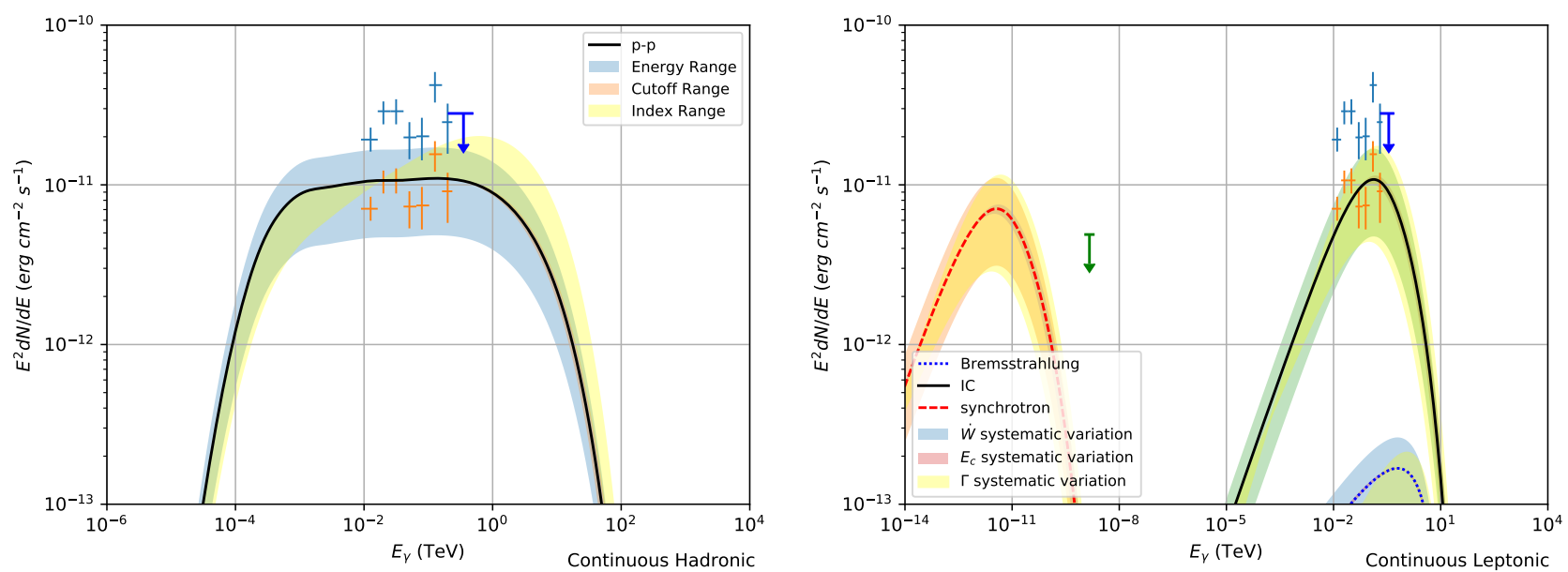

Figure D1. SED example for GeV-A with continuous hadronic SED (left) and continuous leptonic SED (right). This assumes that LS 5039 (at age $10^{5}$ yrs) is the source of acceleration. The upper data points represent the total SED as measured by Fermi-Lat. The lower data points is $36.9 \%$ of this spectra due to source being only GeV-A. The green cross is the ROSAT X-ray upper limit towards GeV-ABC. The blue, pink and yellow shaded regions represent the systematic variation of energy budget $(W)$ or injection luminosity $(\dot{W})$, cutoff energy and spectral index respectively. See Tables 4 and 5 for input parameters. 